\title{
KHOVANOV HOMOLOGY THEORIES AND THEIR APPLICATIONS
}

\author{
ALEXANDER SHUMAKOVITCH \\ To my teacher and advisor, Oleg Yanovich Viro, \\ on the occasion of his $60^{\text {th }}$ birthday
}

\begin{abstract}
This is an expository paper discussing various versions of Khovanov homology theories, interrelations between them, their properties, and their applications to other areas of knot theory and low-dimensional topology.
\end{abstract}

\section{INTRODUCTION}

Khovanov homology is a special case of categorification, a novel approach to construction of knot (or link) invariants that is being actively developed over the last decade after a seminal paper [Kh1] by Mikhail Khovanov. The idea of categorification is to replace a known polynomial knot (or link) invariant with a family of chain complexes, such that the coefficients of the original polynomial are the Euler characteristics of these complexes. Although the chain complexes themselves depend heavily on a diagram that represents the link, their homology depend on the isotopy class of the link only. Khovanov homology categorifies the Jones polynomial [J].

More specifically, let $L$ be an oriented link in $\mathbb{R}^{3}$ represented by a planar diagram $D$ and let $J_{L}(q)$ be a version of the Jones polynomial of $L$ that satisfies the following identities (called the Jones skein relation and normalization):

$$
-q^{-2} J_{+}(q)+q^{2} J_{\beth_{1}}(q)=(q-1 / q) J_{0}(q) ; \quad J \bigcirc(q)=q+1 / q .
$$

The skein relation should be understood as relating the Jones polynomials of three links whose planar diagrams are identical everywhere except in a small disk, where they are different as depicted in (1.1). The normalization fixes the value of the Jones polynomial on the trivial knot. $J_{L}(q)$ is a Laurent polynomial in $q$ for every link $L$ and is completely determined by its skein relation and normalization.

In [Kh1] Mikhail Khovanov assigned to $D$ a family of Abelian groups $\mathcal{H}^{i, j}(L)$ whose isomorphism classes depend on the isotopy class of $L$ only. These groups are defined as homology groups of an appropriate (graded) chain complex $\mathcal{C}^{i, j}(D)$ with integer coefficients. Groups $\mathcal{H}^{i, j}(L)$ are nontrivial for finitely many values of the pair $(i, j)$ only. The gist of the categorification is that the graded Euler characteristic of the Khovanov chain complex equals $J_{L}(q)$ :

$$
J_{L}(q)=\sum_{i, j}(-1)^{i} q^{j} h^{i, j}(L)
$$

where $h^{i, j}(L)=\operatorname{rk}\left(\mathcal{H}^{i, j}(L)\right)$, the Betti numbers of $\mathcal{H}$. The reader is referred to Section 2 for detailed treatment (see also [BN1, Kh1]).

The author is partially supported by NSF grant DMS-0707526. 


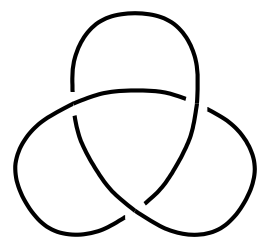

\begin{tabular}{||c||c|c|c|c||}
\hline \hline & 0 & 1 & 2 & 3 \\
\hline \hline 9 & & & & $\mathbf{1}$ \\
\hline 7 & & & & $\mathbf{1}_{\mathbf{2}}$ \\
\hline 5 & & & $\mathbf{1}$ & \\
\hline 3 & $\mathbf{1}$ & & & \\
\hline 1 & $\mathbf{1}$ & & & \\
\hline \hline
\end{tabular}

Figure 1. Right trefoil and its Khovanov homology

In our paper we also make use of another version of the Jones polynomial, denoted $\widetilde{J}_{L}(q)$, that satisfies the same skein relation (1.1) but is normalized to equal 1 on the trivial knot. For the sake of completeness, we also list the skein relation for the original Jones polynomial, $V_{L}(t)$, from $[\mathrm{J}]$ :

$$
t^{-1} V_{+}(t)-t V_{\AA}(t)=\left(t^{1 / 2}-t^{-1 / 2}\right) V_{0}(t) ; \quad V \bigcirc^{(t)=1 .}
$$

We note that $J_{L}(q) \in \mathbb{Z}\left[q, q^{-1}\right]$ while $V_{L}(t) \in \mathbb{Z}\left[t^{1 / 2}, t^{-1 / 2}\right]$. In fact, the terms of $V_{L}(t)$ have half-integer (resp. integer) exponents if $L$ has even (resp. odd) number of components. This is one of the main motivations for our convention (1.1) to be different from (1.3). We also want to ensure that the Jones polynomial of the trivial link has only positive coefficients. The different versions of the Jones polynomial are related as follows:

$$
J_{L}(q)=(q+1 / q) \widetilde{J}_{L}(q), \quad \widetilde{J}_{L}\left(-t^{1 / 2}\right)=V_{L}(t), \quad V_{L}\left(q^{2}\right)=\widetilde{J}_{L}(q)
$$

Another way to look at the Khovanov's identity (1.2) is via the Poincaré polynomial of the Khovanov homology:

$$
K h_{L}(t, q)=\sum_{i, j} t^{i} q^{j} h^{i, j}(L)
$$

With this notation, we get

$$
J_{L}(q)=K h_{L}(-1, q)
$$

1.A. Example. Consider the right trefoil $K$. Its non-zero homology groups are tabulated in Figure 1, where the $i$-grading is represented horizontally and the $j$ grading vertically. The homology is non-trivial for odd $j$-grading only and, hence, even rows are not shown in the table. A table entry of $\mathbf{1}$ or $\mathbf{1}_{\mathbf{2}}$ means that the corresponding group is $\mathbb{Z}$ or $\mathbb{Z}_{2}$, respectively (one can find a more interesting example in Figure 10). In general, an entry of the form $\mathbf{a}, \mathbf{b}_{\mathbf{2}}$ would correspond to the group $\mathbb{Z}^{a} \oplus \mathbb{Z}_{2}^{b}$. For the trefoil $K$, we have that $\mathcal{H}^{0,1}(K) \simeq \mathcal{H}^{0,3}(K) \simeq \mathcal{H}^{2,5}(K) \simeq$ $\mathcal{H}^{3,9}(K) \simeq \mathbb{Z}$ and $\mathcal{H}^{3,7}(K) \simeq \mathbb{Z}_{2}$. Therefore, $K h_{K}(t, q)=q+q^{3}+t^{2} q^{5}+t^{3} q^{9}$. On the other hand, the Jones polynomial of $K$ equals $V_{K}(t)=t+t^{3}-t^{4}$. Relation (1.4) implies that $J_{K}(q)=(q+1 / q)\left(q^{2}+q^{6}-q^{8}\right)=q+q^{3}+q^{5}-q^{9}=K h_{K}(-1, q)$.

Without going into details, we note that the initial categorification of the Jones polynomial by Khovanov was followed with a flurry of activity. Categorifications of 
the colored Jones polynomial $[\mathrm{Kh} 3, \mathrm{BW}]$ and skein $\mathfrak{s l}(3)$ polynomial [Kh4] were based on the original Khovanov's construction. Matrix factorization technique was used to categorify the $\mathfrak{s l}(n)$ skein polynomials [KhR1], HOMFLY-PT polynomial [KhR2], Kauffman polynomial [KhR3], and, more recently, colored $\mathfrak{s l}(n)$ polynomials [Wu, Y]. Ozsváth, Szabó and, independently, Rasmussen used a completely different method of Floer homology to categorify the Alexander polynomial [OS2, Ra1]. Ideas of categorification were successfully applied to tangles, virtual links, skein modules, and polynomial invariants of graphs.

One of the most important recent development in the Khovanov homology theory is the introduction in 2007 of its odd version by Ozsváth, Rasmussen and Szabó [ORS]. The odd Khovanov homology equals the original (even) one modulo 2 and, in particular, categorifies the same Jones polynomial. On the other hand, the odd and even homology theories often have drastically different properties (see Sections 2.4 and 3 for details). The odd Khovanov homology appears to be one of the connecting links between Khovanov and Heegaard-Floer homology theories [OS3].

The importance of the Khovanov homology became apparent after a seminal result by Jacob Rasmussen [Ra2], who used the Khovanov chain complex to give the first purely combinatorial proof of the Milnor conjecture. This conjecture states that the 4-dimensional (slice) genus (and, hence, the genus) of a $(p, q)$-torus knot equals $\frac{(p-1)(q-1)}{2}$. It was originally proved by Kronheimer and Mrowka [KM1] using the gauge theory in 1993.

There are numerous other applications of Khovanov homology theories. They can be used to provide combinatorial proofs of the Slice-Bennequin Inequality and give upper bounds on the Thurston-Bennequin number of Legendrian links, detect quasi-alternating links and find topologically locally-flatly slice knots that are not smoothly slice. We refer the reader to Section 4 for details.

The goal of this paper is to give an overview of the current state of research in Khovanov homology. The exposition is mostly self-contained and no advanced knowledge of the subject is required from the reader. We intentionally limit the scope of our paper to the categorifications of the Jones polynomial only, so as to keep its size under control. The reader is referred to other expository papers on the subject [AKh, Kh6, Ra3] to learn more about the interrelations between different types of categorifications.

We also pay significant attention to experimental aspects of the Khovanov homology. As is often the case with new theories, the initial discovery is led by experiments. It is especially true for Khovanov homology, since it can be computed by hands for a very limited family of knots only. At the moment, there are two programs [BNG, Sh1] that compute Khovanov homology. The first one was written by Dror Bar-Natan and his student Jeremy Green in 2005 and implements the methods from [BN2]. It works significantly faster for knots with sufficiently many crossings (say, more than 15) than the older program KhoHo by the author. On the other hand, KhoHo can compute all the versions of the Khovanov homology that are mentioned in this paper. It is currently the only program that can deal with the odd Khovanov homology. Most of the experimental results that are referred to in this paper were obtained with KhoHo .

This paper is organized as follows. In Section 2 we give a quick overview of constructions involved in the definition of various Khovanov homology theories. We compare these theories with each other and list their basic properties in Section 3. 
Section 4 is devoted to some of the more important applications of the Khovanov homology to other areas of low-dimensional topology.

This paper was originally presented at the Marcus Wallenberg Symposium on Perspectives in Analysis, Geometry, and Topology at Stockholm University in May of 2008. The author would like to thank all the organizers of the Symposium for a very successful and productive meeting. He extends his special thanks to Ilia Itenberg, Burglind Jöricke, and Mikael Passare, the editors of these Proceedings, for their patience with the author. The author is indebted to Mikhail Khovanov for many advises and enlightening discussions during the work on this paper. Finally, the author would like to express his deepest gratitude to Oleg Yanovich Viro for introducing him to the wonderful world of topology 20 years ago and for continuing to be his guide in this world ever since.

\section{Definition of the Khovanov homology}

In this section we give a brief outline of various Khovanov homology theories starting with the original Khovanov's construction. Our setting is slightly more general than the one in the Introduction as we allow different coefficient rings, not only $\mathbb{Z}$.

2.1. Algebraic preliminaries. Let $R$ be a commutative ring with unity. In this paper, we are mainly interested in the cases when $R=\mathbb{Z}, \mathbb{Q}$, or $\mathbb{Z}_{2}$.

2.1.A. Definition. A $\mathbb{Z}$-graded (or simply graded) $R$-module $M$ is an $R$-module decomposed into a direct sum $M=\bigoplus_{j \in \mathbb{Z}} M_{j}$, where each $M_{j}$ is an $R$-module itself. The summands $M_{j}$ are called homogeneous components of $M$ and elements of $M_{j}$ are called the homogeneous elements of degree $j$.

2.1.B. Definition. Let $M=\bigoplus_{j \in \mathbb{Z}} M_{j}$ be a graded free $R$-module. The graded dimension of $M$ is the power series $\operatorname{dim}_{q}(M)=\sum_{j \in \mathbb{Z}} q^{j} \operatorname{dim}\left(M_{j}\right)$ in variable $q$. If $k \in \mathbb{Z}$, the shifted module $M\{k\}$ is defined as having homogeneous components $M\{k\}_{j}=M_{j-k}$.

2.1.C. Definition. Let $M$ and $N$ be two graded $R$-modules. A map $\varphi: M \rightarrow N$ is said to be graded of degree $k$ if $\varphi\left(M_{j}\right) \subset N_{j+k}$ for each $j \in \mathbb{Z}$.

2.1.D. It is an easy exercise to check that $\operatorname{dim}_{q}(M\{k\})=q^{k} \operatorname{dim}_{q}(M), \operatorname{dim}_{q}(M \oplus$ $N)=\operatorname{dim}_{q}(M)+\operatorname{dim}_{q}(N)$, and $\operatorname{dim}_{q}\left(M \otimes_{R} N\right)=\operatorname{dim}_{q}(M) \operatorname{dim}_{q}(N)$, where $M$ and $N$ are graded $R$-modules. Moreover, if $\varphi: M \rightarrow N$ is a graded map of degree $k^{\prime}$, then the shifted map $\varphi: M \rightarrow N\{k\}$ is graded of degree $k^{\prime}+k$. We slightly abuse the notation here by denoting the shifted map in the same way as the map itself.

2.1.E. Definition. Let $(\mathcal{C}, d)=\cdots \longrightarrow \mathcal{C}^{i-1} \stackrel{d^{i-1}}{\longrightarrow} \mathcal{C}^{i} \stackrel{d^{i}}{\longrightarrow} \mathcal{C}^{i+1} \longrightarrow \cdots$ be a (co)chain complex of graded free $R$-modules with graded differentials $d^{i}$ having degree 0 for all $i \in \mathbb{Z}$. Then the graded Euler characteristic of $\mathcal{C}$ is defined as $\chi_{q}(\mathcal{C})=\sum_{i \in \mathbb{Z}}(-1)^{i} \operatorname{dim}_{q}\left(\mathcal{C}^{i}\right)$.

Remark. One can think of a graded (co)chain complex of $R$-modules as a bigraded $R$-module where the homogeneous components are indexed by pairs of numbers $(i, j) \in \mathbb{Z}^{2}$.

Let $A=R[X] / X^{2}$ be the algebra of truncated polynomials. As an $R$-module, $A$ is freely generated by 1 and $X$. We put grading on $A$ by specifying that $\operatorname{deg}(1)=1$ 

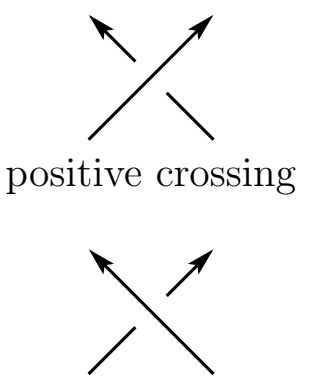

negative crossing

Figure 2. Positive and negative crossings

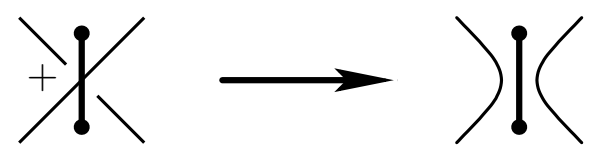

positive marker

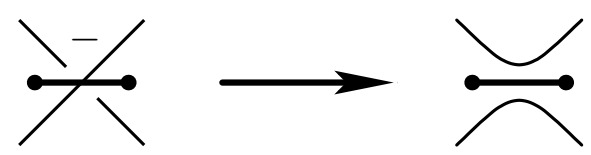

negative marker

and $\operatorname{deg}(X)=-1^{\dagger}$. In other words, $A \simeq R\{1\} \oplus R\{-1\}$ and $\operatorname{dim}_{q}(A)=q+q^{-1}$. At the same time, $A$ is a (graded) commutative algebra with the unity 1 and multiplication $m: A \otimes A \rightarrow A$ given by

$$
m(1 \otimes 1)=1, \quad m(1 \otimes X)=m(X \otimes 1)=X, \quad m(X \otimes X)=0 .
$$

$A$ can also be equipped with a coalgebra structure with comultiplication $\Delta$ : $A \rightarrow A \otimes A$ and counit $\varepsilon: A \rightarrow R$ defined as

$$
\begin{aligned}
\Delta(1) & =1 \otimes X+X \otimes 1, & \Delta(X) & =X \otimes X ; \\
\varepsilon(1) & =0, & \varepsilon(X) & =1 .
\end{aligned}
$$

The comultiplication $\Delta$ is coassociative and cocommutative and satisfies

$$
\begin{aligned}
\left(m \otimes \operatorname{id}_{A}\right) \circ\left(\operatorname{id}_{A} \otimes \Delta\right) & =\Delta \circ m \\
\left(\varepsilon \otimes i_{A}\right) \circ \Delta & =\operatorname{id}_{A}
\end{aligned}
$$

Together with the unit map $\iota: R \rightarrow A$ given by $\iota(1)=1$, this makes $A$ into a commutative Frobenius algebra over $R$ [Kh5].

It follows directly from the definitions that $\iota, \varepsilon, m$, and $\Delta$ are graded maps with

$$
\operatorname{deg}(\iota)=\operatorname{deg}(\varepsilon)=1 \quad \text { and } \quad \operatorname{deg}(m)=\operatorname{deg}(\Delta)=-1 .
$$

2.2. Khovanov chain complex. Let $L$ be an oriented link and $D$ its planar diagram. We assign a number \pm 1 , called sign, to every crossing of $D$ according to the rule depicted in Figure 2. The sum of these signs over all the crossings of $D$ is called the writhe number of $D$ and is denoted by $w(D)$.

Every crossing of $D$ can be resolved in two different ways according to a choice of a marker, which can be either positive or negative, at this crossing (see Figure 3). A collection of markers chosen at every crossing of a diagram $D$ is called a (Kauffman) state of $D$. For a diagram with $n$ crossings, there are, obviously, $2^{n}$ different states. Denote by $\sigma(s)$ the difference between the numbers of positive and negative markers in a given state $s$. Define

$$
i(s)=\frac{w(D)-\sigma(s)}{2}, \quad j(s)=\frac{3 w(D)-\sigma(s)}{2} .
$$

\footnotetext{
${ }^{\dagger}$ We follow the original grading convention from [Kh1] and [BN1] here. It is different by a sign from the one in $[\mathrm{AKh}]$.
} 

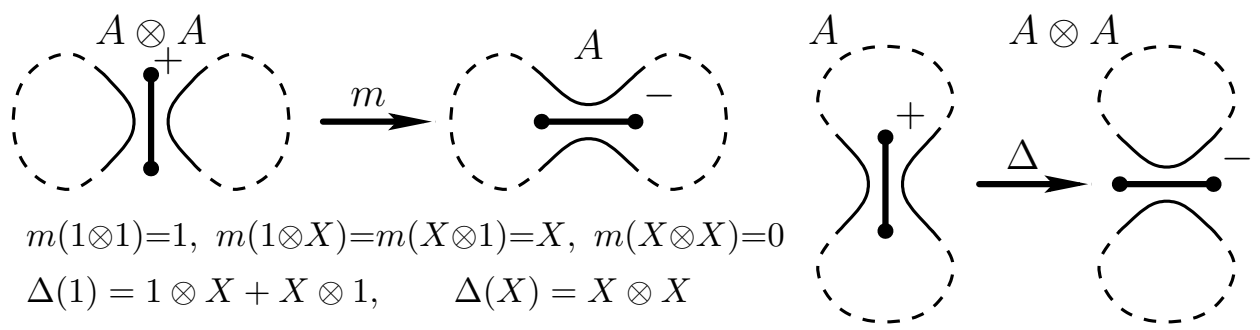

FiguRE 4. Diagram resolutions corresponding to adjacent states and maps between the algebras assigned to the circles

Since both $w(D)$ and $\sigma(s)$ are congruent to $n$ modulo $2, i(s)$ and $j(s)$ are always integer. For a given state $s$, the result of the resolution of $D$ at each crossing according to $s$ is a family $D_{s}$ of disjointly embedded circles. Denote the number of these circles by $\left|D_{s}\right|$.

For each state $s$ of $D$, let $\mathcal{A}(s)=A^{\otimes \mid D_{s}}\{j(s)\}$. One should understand this construction as assigning a copy of algebra $A$ to each circle from $D_{s}$, taking the tensor product of all of these copies, and shifting the grading of the result by $j(s)$. By construction, $\mathcal{A}(s)$ is a graded free $R$-module of graded dimension $\operatorname{dim}_{q}(\mathcal{A}(s))=$ $q^{j(s)}\left(q+q^{-1}\right)^{\left|D_{s}\right|}$. Let $\mathcal{C}^{i}(D)=\bigoplus_{i(s)=i} \mathcal{A}(s)$ for each $i \in \mathbb{Z}$. In order to make $\mathcal{C}(D)$ into a graded complex, we need to define a (graded) differential $d^{i}: \mathcal{C}^{i}(D) \rightarrow$ $\mathcal{C}^{i+1}(D)$ of degree 0. But even before this differential is defined, the (graded) Euler characteristic of $\mathcal{C}(D)$ makes sense.

2.2.A. Lemma. The graded Euler characteristic of $\mathcal{C}(D)$ equals the Jones polynomial of the link L. That is, $\chi_{q}(\mathcal{C}(D))=J_{L}(q)$.

$$
\text { Proof. } \quad \begin{aligned}
\chi_{q}(\mathcal{C}(D)) & =\sum_{i \in \mathbb{Z}}(-1)^{i} \operatorname{dim}_{q}\left(\mathcal{C}^{i}(D)\right) \\
& =\sum_{i \in \mathbb{Z}}(-1)^{i} \sum_{i(s)=i} \operatorname{dim}_{q}(\mathcal{A}(s)) \\
& =\sum_{s}(-1)^{i(s)} q^{j(s)}\left(q+q^{-1}\right)^{\left|D_{s}\right|} \\
& =\sum_{s}(-1)^{\frac{w(D)-\sigma(s)}{2}} q^{\frac{3 w(D)-\sigma(s)}{2}}\left(q+q^{-1}\right)^{\left|D_{s}\right|} .
\end{aligned}
$$

Let us forget for a moment that $A$ denotes an algebra and (temporarily) use this letter for a variable. Substituting $\left(-A^{-2}\right)$ instead of $q$ and noticing that $w(D) \equiv$ $\sigma(s)(\bmod 2)$, we arrive at

$$
\chi_{q}(\mathcal{C}(D))=(-A)^{-3 w(D)} \sum_{s} A^{\sigma(s)}\left(-A^{2}-A^{-2}\right)^{\left|D_{s}\right|}=\left(-A^{2}-A^{-2}\right)\langle L\rangle_{N},
$$

where $\langle L\rangle_{N}$ is the normalized Kauffman bracket polynomial of $L$ (see $[\mathrm{K}]$ for details). The normalized bracket polynomial of a link is related to the bracket polynomial of its diagram as $\langle L\rangle_{N}=(-A)^{-3 w(D)}\langle D\rangle$. Kauffman proved in $[\mathrm{K}]$ that $\langle L\rangle_{N}$ equals the Jones polynomial $V_{L}(t)$ of $L$ after substituting $t^{-1 / 4}$ instead of $A$. The relation (1.4) between $V_{L}(t)$ and $J_{L}(q)$ completes our proof. 
Let $s_{+}$and $s_{-}$be two states of $D$ that differ at a single crossing, where $s_{+}$has a positive marker while $s_{-}$has a negative one. We call two such states adjacent. In this case, $\sigma\left(s_{-}\right)=\sigma\left(s_{+}\right)-2$ and, consequently, $i\left(s_{-}\right)=i\left(s_{+}\right)+1$ and $j\left(s_{-}\right)=$ $j\left(s_{+}\right)+1$. Consider now the resolutions of $D$ corresponding to $s_{+}$and $s_{-}$. One can readily see that $D_{s_{-}}$is obtained from $D_{s_{+}}$by either merging two circles into one or splitting one circle into two (see Figure 4). All the circles that do not pass through the crossing at which $s_{+}$and $s_{-}$differ, remain unchanged. We define $d_{s_{+} s_{-}}: \mathcal{A}\left(s_{+}\right) \rightarrow \mathcal{A}\left(s_{-}\right)$as either $m \otimes$ id or $\Delta \otimes$ id depending on whether the circles merge on split. Here, the multiplication or comultiplication is performed on the copies of $A$ that are assigned to the affected circles, as on Figure 4 , while $d_{s_{+}: s_{-}}$ acts as identity on all the $A$ 's corresponding to the unaffected ones. The difference in grading shift between $\mathcal{A}\left(s_{+}\right)$and $\mathcal{A}\left(s_{-}\right)$and (2.6) ensure that $\operatorname{deg}\left(d_{s_{+}: s_{-}}\right)=0$ by 2.1.D.

We need one more ingredient in order to finish the definition of the differential on $\mathcal{C}(D)$, namely, an ordering of the crossings of $D$. For an adjacent pair of states $\left(s_{+}, s_{-}\right)$, define $\xi\left(s_{+}, s_{-}\right)$to be the number of the negative markers in $s_{+}$(or $s_{-}$) that appear in the ordering of the crossings after the crossing at which $s_{+}$and $s_{-}$ differ. Finally, let $d^{i}=\sum_{\left(s_{+}, s_{-}\right)}(-1)^{\xi\left(s_{+}, s_{-}\right)} d_{s_{+}: s_{-}}$, where $\left(s_{+}, s_{-}\right)$runs over all adjacent pairs of states with $i\left(s_{+}\right)=i$. It is straightforward to verify [Kh1] that $d^{i+1} \circ d^{i}=0$ and, hence, $d: \mathcal{C}(D) \rightarrow \mathcal{C}(D)$ is indeed a differential.

2.2.B. Definition (Khovanov, [Kh1]). The resulting (co)chain complex $\mathcal{C}(D)=$ $\cdots \longrightarrow \mathcal{C}^{i-1}(D) \stackrel{d^{i-1}}{\longrightarrow} \mathcal{C}^{i}(D) \stackrel{d^{i}}{\longrightarrow} \mathcal{C}^{i+1}(D) \longrightarrow \cdots$ is called the Khovanov chain complex of the diagram $D$. The homology of $\mathcal{C}(D)$ with respect to $d$ is called the Khovanov homology of $L$ and is denoted by $\mathcal{H}(L)$. We write $\mathcal{C}(D ; R)$ and $\mathcal{H}(L ; R)$ if we want to emphasize the ring of coefficients that we work with. If $R$ is omitted from the notation, integer coefficients are assumed.

2.2.C. Theorem (Khovanov, [Kh1], see also [BN1]). The isomorphism class of $\mathcal{H}(L ; R)$ depends on the isotopy class of $L$ only and, hence, is a link invariant. In particular, it does not depend on the ordering chosen for the crossings of $D$. $\mathcal{H}(L ; R)$ categorifies $J_{L}(q)$, a version of the Jones polynomial defined by (1.1).

Remark. One can think of $\mathcal{C}(D ; R)$ as a bigraded (co)chain complex $\mathcal{C}^{i, j}(D ; R)$ with a differential of bidegree $(1,0)$. In this case, $i$ is the homological grading of this complex, and $j$ is its $q$-grading, also called the Jones grading. Correspondingly, $\mathcal{H}(L ; R)$ can be considered to be a bigraded $R$-module as well.

2.2.D. Let $\# L$ be the number of components of a link $L$. One can check that $j(s)+\left|D_{s}\right|$ is congruent modulo 2 to $\# L$ for every state $s$. It follows that $\mathcal{C}(D ; R)$ has non-trivial homogeneous components only in the degrees that have the same parity as \#L. Consequently, $\mathcal{H}(L ; R)$ is non-trivial only in the $q$-gradings with this parity (see Example 1.A).

2.2.E. Example. Figure 5 shows the Khovanov chain complex for the Hopf link with the indicated orientation. The diagram has two positive crossings, so its writhe number is 2 . Let $s_{ \pm \pm}$be the four possible resolutions of this diagram, where each "+" or "-" describes the sign of the marker at the corresponding crossings. The chosen ordering of crossings is depicted by numbers placed next to them. By looking at Figure 5, one easily computes that $\mathcal{A}\left(s_{++}\right)=A^{\otimes 2}\{2\}, \mathcal{A}\left(s_{+-}\right)=\mathcal{A}\left(s_{-+}\right)=$ 


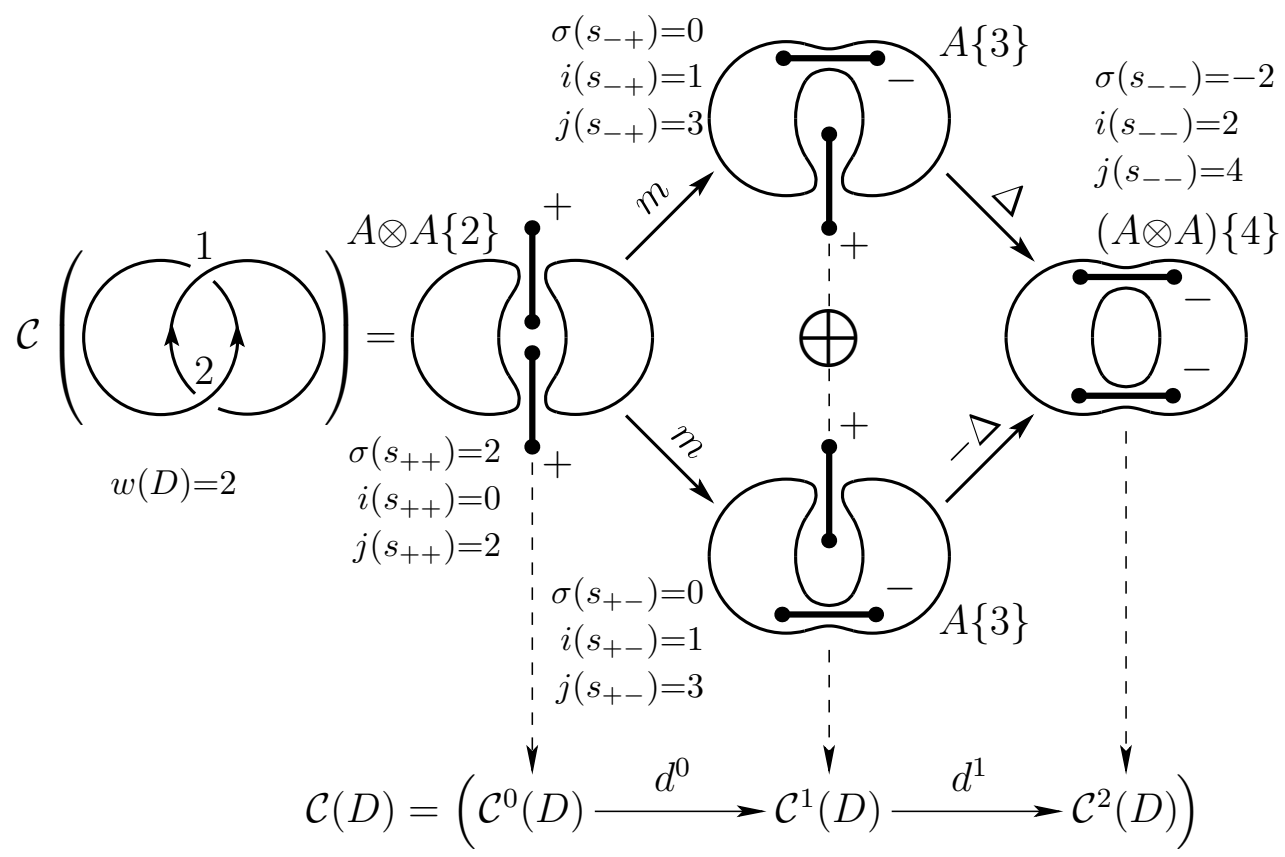

FiguRE 5. Khovanov chain complex for the Hopf link

$A\{3\}$, and $\mathcal{A}\left(s_{--}\right)=A^{\otimes 2}\{4\}$. Correspondingly, $\mathcal{C}^{0}(D)=\mathcal{A}\left(s_{++}\right)=A^{\otimes 2}\{2\}$, $\mathcal{C}^{1}(D)=\mathcal{A}\left(s_{+-}\right) \oplus \mathcal{A}\left(s_{-+}\right)=(A \oplus A)\{3\}$, and $\mathcal{C}^{2}(D)=\mathcal{A}\left(s_{--}\right)=A^{\otimes 2}\{4\}$. It is convenient to arrange the four resolutions in the corners of a square placed in the plane in such a way that its diagonal from $s_{++}$to $s_{--}$is horizontal. Then the edges of this square correspond to the maps between the adjacent states (see Figure 5). We notice that only one of these maps, namely the one corresponding to the edge from $s_{+-}$to $s_{--}$, comes with the negative sign.

In general, $2^{n}$ resolutions of a diagram $D$ with $n$ crossings can be arranged into an $n$-dimensional cube of resolutions, where vertices correspond to the $2^{n}$ states of $D$. The edges of this cube connect adjacent pairs of states and can be oriented from $s_{+}$to $s_{-}$. Every edge is assigned either $m$ or $\Delta$ with the sign $(-1)^{\xi\left(s_{+}, s_{-}\right)}$, as described above. It is easy to check that this makes each square (that is, a 2-dimensional face) of the cube anti-commutative (all squares are commutative without the signs). Finally, the differential $d^{i}$ restricted to each summand $\mathcal{A}(s)$ with $i(s)=i$ equals the sum of all the maps assigned to the edges that originate at $s$.

2.3. Reduced Khovanov homology. Let, as before, $D$ be a diagram of an oriented link $L$. Fix a base point on $D$ that is different from all the crossings. For each state $s$, we define $\widetilde{\mathcal{A}}(s)$ in almost the same way as $\mathcal{A}(s)$, except that we assign $X A$ instead of $A$ to the circle from the resolution $D_{s}$ of $D$ that contains that base point. That is, $\widetilde{\mathcal{A}}(s)=\left((X A) \otimes A^{\otimes\left(\left|D_{s}\right|-1\right)}\right)\{j(s)\}$. We can now build the reduced Khovanov chain complex $\widetilde{\mathcal{C}}(D ; R)$ in exactly the same way as $\mathcal{C}(D ; R)$ by replacing $\mathcal{A}$ with $\widetilde{\mathcal{A}}$ everywhere. The grading shifts and differentials remain the same. It is easy to see that $\widetilde{\mathcal{C}}(D ; R)$ is a subcomplex of $\mathcal{C}(D ; R)$ of index 2 . In fact, it is 
the image of the chain map $\mathcal{C}(D ; R) \rightarrow \mathcal{C}(D ; R)$ that acts by multiplying elements assigned to the circle containing the base point by $X$.

2.3.A. Definition (Khovanov [Kh2], cf. 2.2.B). The homology of $\widetilde{\mathcal{C}}(D ; R)$ is called the reduced Khovanov Homology of $L$ and is denoted by $\widetilde{\mathcal{H}}(L ; R)$. It is clear from the construction of $\widetilde{\mathcal{C}}(D ; R)$ that its graded Euler characteristic equals $\widetilde{J}_{L}(q)$.

2.3.B. TheOREM (Khovanov [Kh2], cf. 2.2.C). The isomorphism class of $\widetilde{\mathcal{H}}(L ; R)$ is a link invariant that categorifies $\widetilde{J}_{L}(q)$, a version of the Jones polynomial defined by (1.1) and (1.4). Moreover, if two base points are chosen on the same component of $L$, then the corresponding reduced Khovanov homologies are isomorphic. On the other hand, $\widetilde{\mathcal{H}}(L ; R)$ might depend on the component of $L$ that the base point is chosen on.

Although $\widetilde{\mathcal{C}}(D ; R)$ can be determined from $\mathcal{C}(D ; R)$, it is in general not clear how $\mathcal{H}(L ; R)$ and $\widetilde{\mathcal{H}}(L ; R)$ are related. There are several examples of pairs of knots (the first one being $14_{9933}^{n}$ and $\overline{15}_{129763}^{n}{ }^{\ddagger}$ ) that have the same rational Khovanov homology, but different rational reduced Khovanov homology. No such examples are known for homologies over $\mathbb{Z}$ among all prime knots with at most 15 crossings. On the other hand, it is proved that $\mathcal{H}\left(L ; \mathbb{Z}_{2}\right)$ and $\widetilde{\mathcal{H}}\left(L ; \mathbb{Z}_{2}\right)$ determine each other completely.

2.3.C. TheOREM $([\operatorname{Sh} 2])$. $\mathcal{H}\left(L ; \mathbb{Z}_{2}\right) \simeq \widetilde{\mathcal{H}}\left(L ; \mathbb{Z}_{2}\right) \otimes_{\mathbb{Z}_{2}} A_{\mathbb{Z}_{2}}$. In particular, $\widetilde{\mathcal{H}}\left(L ; \mathbb{Z}_{2}\right)$ does not depend on the component that the base point is chosen on.

Remark. $X A \simeq R\{0\}$ as a graded $R$-module. It follows that $\widetilde{\mathcal{C}}$ and $\widetilde{\mathcal{H}}$ are nontrivial only in the $q$-gradings with parity different from that of $\# L$, the number of components of $L$ (cf. 2.2.D).

2.4. Odd Khovanov homology. In 2007, Ozsváth, Rasmussen and Szabó introduced [ORS] an odd version of the Khovanov homology. In their theory, the nilpotent variables $X$ assigned to each circle in the resolutions of the link diagram (see Section 2.2) anti-commute rather than commute. The odd Khovanov homology equals the original (even) one modulo 2 and, in particular, categorifies the same Jones polynomial. In fact, the corresponding chain complexes are isomorphic as free bigraded $R$-modules and their differentials are only different by signs. On the other hand, the resulting homology theories often have drastically different properties. We define the odd Khovanov homology below.

Let $L$ be an oriented link and $D$ its planar diagram. To each resolution $s$ of $D$ we assign a free graded $R$-module $\Lambda(s)$ as follows. Label all circles from the resolution $D_{s}$ by some independent variables, say, $X_{1}^{s}, X_{2}^{s}, \ldots, X_{\left|D_{s}\right|}^{s}$ and let $V_{s}=$ $V\left(X_{1}^{s}, X_{2}^{s}, \ldots, X_{\left|D_{s}\right|}^{s}\right)$ be a free $R$-module generated by them. We define $\Lambda(s)=$ $\Lambda^{*}\left(V_{s}\right)$, the exterior algebra of $V_{s}$. Then $\Lambda(s)=\Lambda^{0}\left(V_{s}\right) \oplus \Lambda^{1}\left(V_{s}\right) \oplus \cdots \oplus \Lambda^{\left|D_{s}\right|}\left(V_{s}\right)$ and we grade $\Lambda(s)$ by specifying $\Lambda(s)_{\left|D_{s}\right|-2 k}=\Lambda^{k}\left(V_{s}\right)$ for each $0 \leq k \leq\left|D_{s}\right|$, where $\Lambda(s)_{\left|D_{s}\right|-2 k}$ is the homogeneous component of $\Lambda(s)$ of degree $\left|D_{s}\right|-2 k$. It is an easy exercise for the reader to check that $\operatorname{dim}_{q}(\Lambda(s))=\operatorname{dim}_{q}\left(A^{\otimes\left|D_{s}\right|}\right)$.

Just as in the case of the even Khovanov homology, these $R$-modules $\Lambda(s)$ can be arranged into an $n$-dimensional cube of resolutions. Let $\mathcal{C}_{\text {odd }}^{i}(D)=\bigoplus_{i(s)=i} \Lambda(s)\{j(s\}$.

\footnotetext{
${ }^{\ddagger}$ Here, $14_{9933}^{n}$ denotes the non-alternating knot number 9933 with 14 crossings from the Knotscape knot table [HTh] and $\overline{15}_{129763}^{n}$ is the mirror image of the knot $15_{129763}^{n}$. See also remark on page 13 .
} 


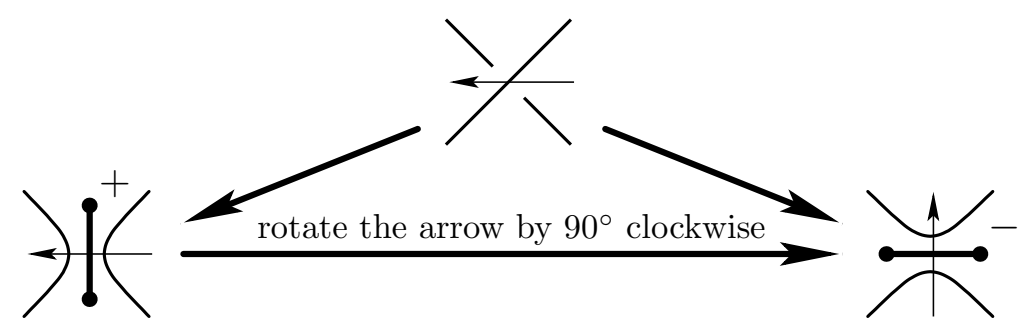

FiguRE 6. Choice of arrows at the diagram crossings

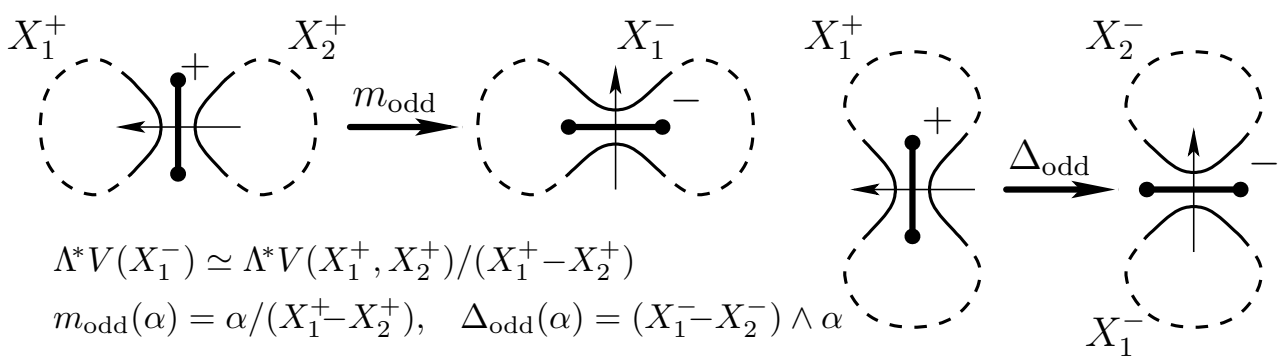

Figure 7. Adjacent states and differentials in the odd Khovanov chain complex

Then, similarly to Lemma 2.2.A, we have that $\chi_{q}\left(\mathcal{C}_{\text {odd }}(D)\right)=J_{L}(q)$. In fact, $\mathcal{C}_{\text {odd }}(D) \simeq \mathcal{C}(D)$ as bigraded $R$-modules. In order to define the differential on $\mathcal{C}_{\text {odd }}$, we need to introduce an additional structure, a choice of an arrow at each crossing of $D$ that is parallel to the negative marker at that crossing (see Figure 6). There are obviously $2^{n}$ such choices. For every state $s$ on $D$, we place arrows that connect two branches of $D_{s}$ near each (former) crossing according to the rule from Figure 6 .

We now assign (graded) maps $m_{\text {odd }}$ and $\Delta_{\text {odd }}$ to each edge of the cube of resolutions that connects adjacent states $s_{+}$and $s_{-}$. If $s_{-}$is obtained from $s_{+}$by merging two circles together, then $\Lambda\left(s_{-}\right) \simeq \Lambda\left(s_{+}\right) /\left(X_{1}^{+}-X_{2}^{+}\right)$, where $X_{1}^{+}$and $X_{2}^{+}$ are the generators of $V_{s_{+}}$corresponding to the two merging circles, as depicted in Figure 7 . We define $m_{\text {odd }}: \Lambda\left(s_{+}\right) \rightarrow \Lambda\left(s_{-}\right)$to be this isomorphism composed with the projection $\Lambda\left(s_{+}\right) \rightarrow \Lambda\left(s_{+}\right) /\left(X_{1}^{+}-X_{2}^{+}\right)$.

The case when one circle splits into two is more interesting. Let $X_{1}^{-}$and $X_{2}^{-}$be the generators of $V_{s_{-}}$corresponding to these two circles such that the arrow points from $X_{1}^{-}$to $X_{2}^{-}$(see Figure 7 ). Now for each generator $X_{k}^{+}$of $V_{s_{+}}$, we define $\Delta_{\text {odd }}\left(X_{k}^{+}\right)=\left(X_{1}^{-}-X_{2}^{-}\right) \wedge X_{\eta(k)}^{-}$where $\eta$ is the correspondence between circles in $D_{s_{+}}$and $D_{s_{-}}$. While $\eta(1)$ can equal either 1 or 2 , this choice does not affect $\Delta_{\text {odd }}\left(X_{1}^{+}\right)$since $\left(X_{1}^{-}-X_{2}^{-}\right) \wedge X_{2}^{-}=X_{1}^{-} \wedge X_{2}^{-}=-X_{2}^{-} \wedge X_{1}^{-}=\left(X_{1}^{-}-X_{2}^{-}\right) \wedge X_{1}^{-}$.

This definition makes each square in the cube of resolutions either commutative, or anti-commutative, or both. The latter case means that both double-composites corresponding to the square are trivial. This is a major departure from the situation that we had in the even case, where each square was commutative. In particular, it makes the choice of signs on the edges of the cube much more involved.

2.4.A. Theorem (Ozsváth-Rasmussen-Szabó [ORS]). It is possible to assign a sign to each edge in this (odd) cube of resolutions in such a way that every square 


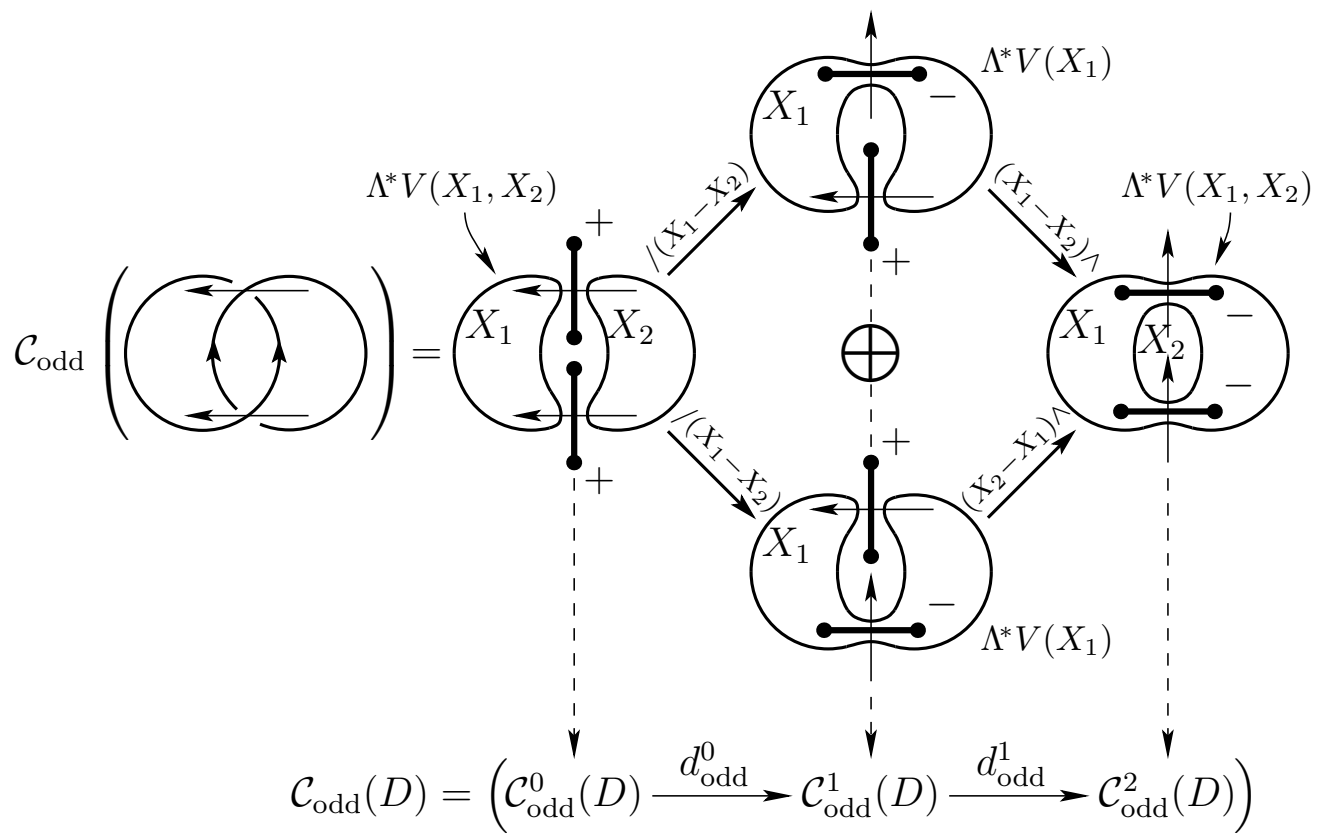

Figure 8. Odd Khovanov chain complex for the Hopf link

becomes anti-commutative. This results in a graded (co)chain complex $\mathcal{C}_{\text {odd }}(D ; R)$. The homology $\mathcal{H}_{\text {odd }}(L ; R)$ of $\mathcal{C}_{\text {odd }}(D ; R)$ does not depend on the choice of arrows at the crossings, the choice of edge signs, and some other choices needed in the construction. Moreover, the isomorphism class of $\mathcal{H}_{\mathrm{odd}}(L ; R)$ is a link invariant, called odd Khovanov homology, that categorifies $J_{L}(q)$.

Remark. There is no explicit construction for assigning signs to the edges of the cube of resolutions in the case of the odd Khovanov chain complex. The Theorem above only ensures that signs exist.

2.4.B. By comparing the definitions of $\mathcal{C}_{\text {odd }}\left(D ; \mathbb{Z}_{2}\right)$ and $\mathcal{C}\left(D ; \mathbb{Z}_{2}\right)$, it is easy to see that they are isomorphic as graded chain complexes (since the signs do not matter modulo 2). It follows that $\mathcal{H}_{\text {odd }}\left(D ; \mathbb{Z}_{2}\right) \simeq \mathcal{H}\left(D ; \mathbb{Z}_{2}\right)$ as well.

2.4.C. One can construct reduced odd Khovanov chain complex $\widetilde{\mathcal{C}}_{\text {odd }}(D ; R)$ and reduced odd Khovanov homology $\widetilde{\mathcal{H}}_{\text {odd }}(L ; R)$ using methods similar to those from Sections 2.3. In this case, contrary to the even situation, reduced and non-reduced odd Khovanov homology determine each other completely (see [ORS]). Namely, $\mathcal{H}_{\text {odd }}(L ; R) \simeq \widetilde{\mathcal{H}}_{\text {odd }}(L ; R)\{1\} \oplus \widetilde{\mathcal{H}}_{\text {odd }}(L ; R)\{-1\}$ (cf. Theorem 2.3.C). It is therefore enough to consider the reduced version of the odd Khovanov homology only.

2.4.D. Example. The odd Khovanov chain complex for the Hopf link is depicted in Figure 8. All the grading shifts in this case are the same as in Example 2.2.E and on Figure 5, so we do not list them again. We notice that the resulting square of resolutions is anti-commutative, so no adjustment of signs is needed. 
The odd Khovanov homology should provide an insight into interrelations between Khovanov and Heegaard-Floer [OS1] homology theories. Its definition was motivated by the following result.

2.4.E. TheOrem (Ozsváth-Szabó [OS3]). For each link L with a diagram D, there exists a spectral sequence with $E^{1}=\widetilde{\mathcal{C}}\left(D ; \mathbb{Z}_{2}\right)$ and $E^{2}=\widetilde{\mathcal{H}}\left(L ; \mathbb{Z}_{2}\right)$ that converges to the $\mathbb{Z}_{2}$-Heegaard-Floer homology $\widehat{H F}\left(\Sigma(L) ; \mathbb{Z}_{2}\right)$ of the double branched cover $\Sigma(L)$ of $S^{3}$ along $L$.

2.4.F. CONJECTURE. There exists a spectral sequence that starts with $\widetilde{\mathcal{C}}_{\text {odd }}(D ; \mathbb{Z})$ and $\widetilde{\mathcal{H}}_{\text {odd }}(L ; \mathbb{Z})$ and converges to $\widehat{H F}(\Sigma(L) ; \mathbb{Z})$.

\section{Properties of the Khovanov homology}

In this section we summarize main properties of the Khovanov homology and list related constructions. We emphasize similarities and differences in properties exhibited by different versions of the Khovanov homology. Some of them were already mentioned in the previous sections.

3.A. Let $L$ be an oriented link and $D$ its planar diagram. Then

- $\widetilde{\mathcal{C}}(D ; R)$ is a subcomplex of $\mathcal{C}(D ; R)$ of index 2 .

- $\mathcal{H}_{\text {odd }}\left(L ; \mathbb{Z}_{2}\right) \simeq \mathcal{H}\left(L ; \mathbb{Z}_{2}\right)$ and $\widetilde{\mathcal{H}}_{\text {odd }}\left(L ; \mathbb{Z}_{2}\right) \simeq \widetilde{\mathcal{H}}\left(L ; \mathbb{Z}_{2}\right)$.

- $\chi_{q}(\mathcal{H}(L ; R))=\chi_{q}\left(\mathcal{H}_{\text {odd }}(L ; R)\right)=J_{L}(q)$ and $\chi_{q}(\widetilde{\mathcal{H}}(L ; R))=\chi_{q}\left(\widetilde{\mathcal{H}}_{\text {odd }}(L ; R)\right)=\widetilde{J}_{L}(q)$.

- $\mathcal{H}\left(L ; \mathbb{Z}_{2}\right) \simeq \widetilde{\mathcal{H}}\left(L ; \mathbb{Z}_{2}\right) \otimes_{\mathbb{Z}_{2}} A_{\mathbb{Z}_{2}}\left[\right.$ Sh2] and $\mathcal{H}_{\text {odd }}(L ; R) \simeq \widetilde{\mathcal{H}}_{\text {odd }}(L ; R)\{1\} \oplus$ $\widetilde{\mathcal{H}}_{\text {odd }}(L ; R)\{-1\}[\mathrm{ORS}]$. On the other hand, $\mathcal{H}(L ; \mathbb{Z})$ and $\mathcal{H}(L ; \mathbb{Q})$ do not split in general.

- For links, $\widetilde{\mathcal{H}}\left(L ; \mathbb{Z}_{2}\right)$ and $\widetilde{\mathcal{H}}_{\text {odd }}(L ; R)$ do not depend on the choice of a component with the base point. This is, in general, not the case for $\widetilde{\mathcal{H}}(L ; \mathbb{Z})$ and $\widetilde{\mathcal{H}}(L ; \mathbb{Q})$.

- If $L$ is a non-split alternating link, then $\mathcal{H}(L ; \mathbb{Q}), \widetilde{\mathcal{H}}(L ; R)$, and $\widetilde{\mathcal{H}}_{\text {odd }}(L ; R)$ are completely determined by the Jones polynomial and signature of $L[\mathrm{Kh} 2$, L, ORS].

- $\mathcal{H}\left(L ; \mathbb{Z}_{2}\right)$ and $\mathcal{H}_{\text {odd }}(L ; \mathbb{Z})$ are invariant under the component-preserving link mutations $[\mathrm{B}, \mathrm{W} 2]$. It is unclear whether the same holds true for $\mathcal{H}(L ; \mathbb{Z})$. On the other hand, $\mathcal{H}(L ; \mathbb{Z})$ is known not to be preserved under a mutation that exchanges components of a link [W1] and under a cabled mutation [DGShT].

- $\mathcal{H}(L ; \mathbb{Z})$ almost always has torsion (except for several special cases), but mostly of order 2. The first knot with 4-torsion is the $(4,5)$-torus knot that has 15 crossings. The first known knot with 3 -torsion is the $(5,6)$-torus knot with 24 crossings. On the other hand, $\mathcal{H}_{\text {odd }}(L ; \mathbb{Z})$ was observed to have torsion of various orders even for knots with relatively few crossings (see remark on page 21), although orders 2 and 3 are the most popular.

- $\widetilde{\mathcal{H}}(L ; \mathbb{Z})$ has very little torsion. The first knot with torsion has 13 crossings. On the other hand, $\widetilde{\mathcal{H}}_{\text {odd }}(L ; \mathbb{Z})$ has as much torsion as $\mathcal{H}_{\text {odd }}(L ; \mathbb{Z})$.

Remark. The properties above show that $\widetilde{\mathcal{H}}_{\text {odd }}(L ; \mathbb{Z})$ behaves similarly to $\widetilde{\mathcal{H}}\left(L ; \mathbb{Z}_{2}\right)$ but not to $\widetilde{\mathcal{H}}(L ; \mathbb{Z})$. This is by design (see 2.4.E and 2.4.F). 


\subsection{Homological thickness.}

3.1.A. Definition. Let $L$ be a link. The homological width of $L$ over a ring $R$ is the minimal number of adjacent diagonals $j-2 i=$ const such that $\mathcal{H}(L ; R)$ is zero outside of these diagonals. It is denoted by $\mathrm{hw}_{R}(L)$. The reduced homological width, $\widetilde{\mathrm{hw}}_{R}(L)$ of $L$, odd homological width, $\mathrm{ohw}_{R}(L)$ of $L$, and reduced odd homological width, $\widetilde{\mathrm{ohw}}_{R}(L)$ of $L$ are defined similarly.

3.1.B. It follows from 2.4.C that $\widetilde{\mathrm{ohw}}_{R}(L)=\mathrm{ohw}_{R}(L)-1$. The same holds true in the case of the even Khovanov homology over $\mathbb{Q}: \widetilde{h_{\mathbb{Q}}}(L)=\operatorname{hw}_{\mathbb{Q}}(L)-1($ see $[\mathrm{Kh} 2])$.

3.1.C. Definition. A link $L$ is said to be homologically thin over a ring $R$, or simply $R \mathrm{H}$-thin, if $\mathrm{hw}_{R}(L)=2$. $L$ is homologically thick, or $R \mathrm{H}$-thick, otherwise. We define odd-homologically thin and thick, or simply $R \mathrm{OH}$-thin and $R \mathrm{OH}$-thick, links similarly.

3.1.D. Theorem (Lee, Ozsváth-Rasmussen-Szabó, Manolescu-Ozsváth [L, ORS, $\mathrm{MO}]$ ). Quasi-alternating links (see Section 4.3 for the definition) are $\mathrm{RH}$-thin and $\mathrm{ROH}$-thin for every ring $\mathrm{R}$. In particular, this is true for non-split alternating links.

3.1.E. TheOREm (Khovanov [Kh2]). Adequate links are $R H$-thick for every $R$.

3.1.F. Homological thickness of a link $L$ often does not depend on the base ring. The first prime knot with $\mathrm{hw}_{\mathbb{Q}}(L)<\mathrm{hw}_{\mathbb{Z}_{2}}(L)$ and $\mathrm{hw}_{\mathbb{Q}}(L)<\mathrm{hw}_{\mathbb{Z}}(L)$ is $15_{41127}^{n}$ with 15 crossings (see Figure 9). The first prime knot that is $\mathbb{Q H}$-thin but $\mathbb{Z H}$ thick, $16_{197566}^{n}$, has 16 crossings (see Figure 10). Its mirror image, $\overline{16}_{197566}^{n}$ is both $\mathbb{Q H}$ - and $\mathbb{Z}$ H-thin but is $\mathbb{Z}_{2} \mathrm{H}$-thick with $\mathcal{H}^{-8,-21}\left(\overline{16}_{197566}^{n} ; \mathbb{Z}_{2}\right) \simeq \mathbb{Z}_{2}$, for example, because of the Universal Coefficient Theorem. Also observe that $\mathcal{H}^{9,25}\left(16_{197566}^{n} ; \mathbb{Z}\right)$ and $\mathcal{H}^{-8,-25}\left(\overline{16}_{197566}^{n} ; \mathbb{Z}\right)$ have 4 -torsion, shown in a small box in the tables.

Remark. Throughout this paper we use the following notation for knots: knots with 10 crossings or less are numbered according to the Rolfsen's knot table [Ro] and knots with 11 crossings or more are numbered according to the knot table from Knotscape [HTh]. Mirror images of knots from either table are denoted with a bar on top. For example, $\overline{9}_{46}$ is the mirror image of the knot number 46 with 9 crossings from the Rolfsen's table and $16_{197566}^{n}$ is the non-alternating knot number 197566 with 16 crossings from the Knotscape's one.

3.1.G. Odd Khovanov homology is often thicker over $\mathbb{Z}$ than the even one. This is crucial for applications (see Section 4). On the other hand, $\widetilde{\operatorname{ohw}_{\mathbb{Q}}}(L) \leq \widetilde{\mathrm{hw}_{\mathbb{Q}}}(L)$ for all but one prime knot with at most 15 crossings. The homology for this knot, $15_{41127}^{n}$, is shown in Figure 9. Please observe that $\widetilde{\mathcal{H}}_{\text {odd }}\left(15_{41127}^{n}\right)$ has 3 -torsion (in gradings $(-2,-2)$ and $(-1,0))$, while $\widetilde{\mathcal{H}}\left(15_{41127}^{n}\right)$ has none.

3.2. Lee spectral sequence and the Knight-Move Conjecture. In [L] Eun Soo Lee introduced a structure of a spectral sequence on the rational Khovanov chain complex $\mathcal{C}(D ; \mathbb{Q})$ of a link diagram $D$. Namely, Lee defined a differential $d^{\prime}: \mathcal{C}(D ; \mathbb{Q}) \rightarrow \mathcal{C}(D ; \mathbb{Q})$ of bidegree $(1,4)$ by setting

$$
\begin{array}{ll}
m^{\prime}: A \otimes A \rightarrow A: & m^{\prime}(1 \otimes 1)=m^{\prime}(1 \otimes X)=m^{\prime}(X \otimes 1)=0, \quad m^{\prime}(X \otimes X)=1 \\
\Delta^{\prime}: A \rightarrow A \otimes A: & \Delta^{\prime}(1)=0, \quad \Delta^{\prime}(X)=1 \otimes 1
\end{array}
$$




\begin{tabular}{|r||l|l|l|l|l|l|l|l|l|l|l||}
\hline \hline & -7 & -6 & -5 & -4 & -3 & -2 & -1 & 0 & 1 & 2 & 3 \\
\hline \hline 8 & & & & & & & & & & & $\mathbf{1}$ \\
\hline 6 & & & & & & & & & & $\mathbf{1}$ & \\
\hline 4 & & & & & & & & & $\mathbf{1}$ & & \\
\hline 2 & & & & & & & $\mathbf{1}$ & $\mathbf{2}$ & & & \\
\hline 0 & & & & & & $\mathbf{1}$ & & $\mathbf{1} \mathbf{2}$ & & & \\
\hline-2 & & & & & $\mathbf{1}$ & $\mathbf{1}$ & & & & & \\
\hline-4 & & & & $\mathbf{2}$ & $\mathbf{1}$ & & & & & & \\
\hline-6 & & & $\mathbf{1}$ & & & & & & & & \\
\hline-8 & & $\mathbf{1}$ & & & & & & & & & \\
\hline-10 & $\mathbf{1}$ & & & & & & & & & & \\
\hline \hline
\end{tabular}

$$
\mathrm{hw}_{\mathbb{Q}}=3, \quad \widetilde{\mathrm{hw}_{\mathbb{Q}}}=2, \quad \mathrm{hw}_{\mathbb{Z}}=4, \quad \widetilde{\mathrm{hw}_{\mathbb{Z}}}=3
$$

\begin{tabular}{|r||r|r|r|r|r|r|r|r|r|r|r||}
\hline \hline & -7 & -6 & -5 & -4 & -3 & -2 & -1 & 0 & 1 & 2 & 3 \\
\hline \hline 8 & & & & & & & & & & & $\mathbf{1}$ \\
\hline 6 & & & & & & & & & & $\mathbf{1}$ & \\
\hline 4 & & & & & & & & & $\mathbf{1}$ & & \\
\hline 2 & & & & & & & & $\mathbf{1}, \mathbf{1}_{\mathbf{2}}$ & & & \\
\hline 0 & & & & & & & $\mathbf{\mathbf { 1 } _ { \mathbf { 6 } }}$ & $\mathbf{1}$ & & & \\
\hline-2 & & & & & & $\mathbf{\mathbf { 1 } _ { 6 }}$ & & & & & \\
\hline-4 & & & & $\mathbf{1}$ & $\mathbf{1}_{\mathbf{2}}$ & & & & & & \\
\hline-6 & & & $\mathbf{1}$ & & & & & & & & \\
\hline-8 & & $\mathbf{1}$ & & & & & & & & & \\
\hline-10 & $\mathbf{1}$ & & & & & & & & & & \\
\hline \hline
\end{tabular}

$$
\operatorname{ohw}_{\mathbb{Q}}=4, \quad \widetilde{\operatorname{ohw}_{\mathbb{Q}}}=3, \quad \operatorname{ohw}_{\mathbb{Z}}=4, \quad \widetilde{\operatorname{ohw}}_{\mathbb{Z}}=3
$$

Figure 9. Integral reduced even Khovanov homology (above) and odd Khovanov Homology (below) of the knot $15_{41127}^{n}$

It is straightforward to verify that $d^{\prime}$ is indeed a differential and that it anticommutes with $d$, that is $d \circ d^{\prime}+d^{\prime} \circ d=0$. This makes $\left(\mathcal{C}(D ; \mathbb{Q}), d, d^{\prime}\right)$ into a double complex. Let $d_{*}^{\prime}$ be the differential induced by $d^{\prime}$ on $\mathcal{H}(L ; \mathbb{Q})$. Lee proved that $d_{*}^{\prime}$ is functorial, that is, it commutes with isomorphisms induced on $\mathcal{H}(L ; \mathbb{Q})$ by isotopies of $L$. It follows that there exists a spectral sequence with $\left(E_{1}, d_{1}\right)=(\mathcal{C}(D ; \mathbb{Q}), d)$ 


\begin{tabular}{|c|c|c|c|c|c|c|c|c|c|c|c|c|c|}
\hline & -2 & -1 & 0 & 1 & 2 & 3 & 4 & 5 & 6 & 7 & 8 & 9 & 10 \\
\hline 29 & & & & & & & & & & & & & 1 \\
\hline 27 & & & & & & & & & & & & 4 & $\mathbf{1}_{2}$ \\
\hline 25 & & & & & & & & & & & 7 & $1,3_{2} 1_{4}$ & \\
\hline 23 & & & & & & & & & & 12 & $4,8_{2}$ & & \\
\hline 21 & & & & & & & & & 15 & $7,12_{2}$ & $1_{2}$ & & \\
\hline 19 & & & & & & & & 17 & $12,16_{2}$ & & & & \\
\hline 17 & & & & & & & 16 & $15,18_{2}$ & $1_{2}$ & & & & \\
\hline 15 & & & & & & 15 & $17,16_{2}$ & $1_{2}$ & & & & & \\
\hline 13 & & & & & 10 & $16,15_{2}$ & & & & & & & \\
\hline 11 & & & & 6 & $15,10_{2}$ & & & & & & & & \\
\hline 9 & & & 3 & $10,6_{2}$ & & & & & & & & & \\
\hline 7 & & 1 & $7,2_{2}$ & & & & & & & & & & \\
\hline 5 & & $2,1_{2}$ & & & & & & & & & & & \\
\hline 3 & 1 & & & & & & & & & & & & \\
\hline
\end{tabular}

The free part of $\mathcal{H}\left(16_{197566}^{n} ; \mathbb{Z}\right)$ is supported on diagonals $j-2 i=7$ and $j-2 i=9$. On the other hand, there is 2 -torsion on the diagonal $j-2 i=5$. Therefore, $16_{197566}^{n}$ is $\mathbb{Q H}$-thin, but $\mathbb{Z} \mathrm{H}$-thick and $\mathbb{Z}_{2} \mathrm{H}$-thick.

\begin{tabular}{|c|c|c|c|c|c|c|c|c|c|c|c|c|c|}
\hline & -10 & -9 & -8 & -7 & -6 & -5 & -4 & -3 & -2 & -1 & 0 & 1 & 2 \\
\hline-3 & & & & & & & & & & & & & 1 \\
\hline-5 & & & & & & & & & & & & 2 & $1_{2}$ \\
\hline-7 & & & & & & & & & & & 7 & $1,2_{2}$ & \\
\hline-9 & & & & & & & & & & 10 & $3,6_{2}$ & & \\
\hline-11 & & & & & & & & & 15 & $6,10_{2}$ & & & \\
\hline-13 & & & & & & & & 16 & $10,15_{2}$ & & & & \\
\hline-15 & & & & & & & $17,1_{2}$ & $15,16_{2}$ & & & & & \\
\hline-17 & & & & & & $15,1_{2}$ & $16,18_{2}$ & & & & & & \\
\hline-19 & & & & & 12 & $17,16_{2}$ & & & & & & & \\
\hline-21 & & & & $7,1_{2}$ & $15,12_{2}$ & & & & & & & & \\
\hline-23 & & & 4 & $12,8_{2}$ & & & & & & & & & \\
\hline-25 & & 1 & $7,3_{2} \mathbf{1}_{4}$ & & & & & & & & & & \\
\hline-27 & & $4,1_{2}$ & & & & & & & & & & & \\
\hline-29 & 1 & & & & & & & & & & & & \\
\hline
\end{tabular}

$\mathcal{H}\left(\overline{16}_{197566}^{n} ; \mathbb{Z}\right)$ is supported on diagonals $j-2 i=-7$ and $j-2 i=-9$.

But there is 2-torsion on the diagonal $j-2 i=-7$.

Therefore, $\overline{16}_{197566}^{n}$ is $\mathbb{Q H}$-thin and $\mathbb{Z} \mathrm{H}$-thin, but $\mathbb{Z}_{2} \mathrm{H}$-thick.

FiguRE 10. Integral Khovanov homology of the knots $16_{197566}^{n}$ and $\overline{16}_{197566}^{n}$ 
and $\left(E_{2}, d_{2}\right)=\left(\mathcal{H}(L ; \mathbb{Q}), d_{*}^{\prime}\right)$ that converges to the homology of the total (filtered) complex of $\mathcal{C}(D ; \mathbb{Q})$ with respect to the differential $d+d^{\prime}$. It is called the Lee spectral sequence. The differentials $d_{n}$ in this spectral sequence have bidegree $(1,4(n-1))$.

3.2.A. Theorem (Lee $[\mathrm{L}])$. If $L$ is an oriented link with $\# L$ components, then $H\left(\operatorname{Total}(\mathcal{C}(D ; \mathbb{Q})), d+d^{\prime}\right)$, the limit of the Lee spectral sequence, consists of $2^{n-1}$ copies of $\mathbb{Q} \oplus \mathbb{Q}$, each located in a specific homological grading that is explicitly defined by linking numbers of the components of $L$. In particular, if $L$ is a knot, then the Lee spectral sequence converges to $\mathbb{Q} \oplus \mathbb{Q}$ localed in homological grading 0 .

The following Theorem is the cornerstone in the definition of the Rasmussen invariant, one of the main applications of the Khovanov homology (see Section 4.1).

3.2.B. Theorem (Rasmussen $[\mathrm{Ra} 2])$. If $L$ is a knot, then the two copies of $\mathbb{Q}$ in the limiting term of the Lee spectral sequence for $L$ are "neighbors", that is, their q-gradings are different by 2 .

3.2.C. Corollary. If the Lee spectral sequence for a link $L$ collapses after the second page (that is, $d_{n}=0$ for $n \geq 3$ ), then $\mathcal{H}(L ; \mathbb{Q})$ consists of one "pawn-move" pair in homological grading 0 and multiple "knight-move" pairs, shown below, with appropriate grading shifts.

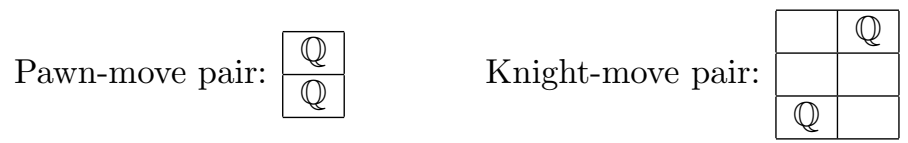

3.2.D. Corollary. Since $d_{3}$ has bidegree $(1,8)$, the Lee spectral sequence collapses after the second page for all knots with homological width 2 or 3 , in particular, for all alternating and quasi-alternating knots. Hence, Corollary 3.2.C can be applied to such knots.

3.2.E. Knight-Move Conjecture (Garoufalidis-Khovanov-Bar-Natan [BN1, Kh1]). The conclusion of Corollary 3.2.C is true for every knot.

Remark. There are currently no known counter-examples to the Knight-Move Conjecture. In fact, the Lee spectral sequence can be proved (in one way or another) to collapse after the second page for every known example of Khovanov homology.

Remark. While the Lee spectral sequence exists over any ring $R$, the statement of Theorem 3.2.A does not hold true for all of them. In particular, it is wrong over $\mathbb{Z}_{2}$. In this case, though, a similar theory was constructed by Paul Turner $[\mathrm{T}]$. In fact, his construction works for reduced Khovanov homology as well because of 2.3.C. While Theorems 3.2.A and 3.2.B are still true over $\mathbb{Z}_{p}$ with odd prime $p$, Knight-Move Conjecture is known to be false over such rings [BN2].

Remark. The Lee spectral sequence has no analog in the odd and reduced Khovanov homology theories (except over $\mathbb{Z}_{2}$, as noted above, where the two theories coincide). The Knight-Move Conjecture has no analog in these theories either.

3.3. Long exact sequence of the Khovanov homology. One of the most useful tools in studying Khovanov homology is the long exact sequence that categorifies the Kauffman's unoriented skein relation for the Jones polynomial [Kh1]. If we forget about the grading, then it is clear from the construction from Section 2.2 that $\mathcal{C}(\asymp)$ is a subcomplex of $\mathcal{C}(久)$ and $\mathcal{C}(\rangle) \simeq \mathcal{C}(久) / \mathcal{C}(\asymp)$ (see also Figure 5). Here, 
$\asymp$ and ) ( depict link diagrams where a single crossing $久$ is resolved in a negative or, respectively, positive direction. This results in a short exact sequence of non-graded chain complexes:

$$
0 \longrightarrow \mathcal{C}(\asymp) \stackrel{\text { in }}{\longrightarrow} \mathcal{C}(\text { ( }) \stackrel{p}{\longrightarrow} \mathcal{C}(\rangle) \longrightarrow 0,
$$

where in is the inclusion and $p$ is the projection.

In order to introduce grading into (3.2), we need to consider the cases when the crossing to be resolved is either positive or negative. We get (see [Ra3]):

$$
\begin{aligned}
& \left.0 \longrightarrow \mathcal{C}(\asymp)\{2+3 \omega\}[1+\omega] \stackrel{\text { in }}{\longrightarrow} \mathcal{C}(\stackrel{\Upsilon}{\complement}) \stackrel{p}{\longrightarrow} \mathcal{C}()^{\prime}\right)\{1\} \longrightarrow 0, \\
& 0 \longrightarrow \mathcal{C}()(-1\} \stackrel{\text { in }}{\longrightarrow} \mathcal{C}\left(\bigwedge^{\searrow} \stackrel{p}{\longrightarrow} \mathcal{C}(\asymp)\{1+3 \omega\}[\omega] \longrightarrow 0,\right.
\end{aligned}
$$

where $\omega$ is the difference between the numbers of negative crossings in the unoriented resolution $\asymp$ (it has to be oriented somehow in order to define its Khovanov chain complex) and in the original diagram. The notation $\mathcal{C}[k]$ is used to represent a shift in the homological grading of a complex $\mathcal{C}$ by $k$. The graded versions of in and $p$ are both homogeneous, that is, have bidegree $(0,0)$.

By passing to homology in (3.3), we get the following result.

3.3.A. Theorem (Khovanov, Viro, Rasmussen [Kh1, V, Ra3]). The Khovanov homology is subject to the following long exact sequences:

$$
\begin{aligned}
& \cdots \longrightarrow \mathcal{H}(\rangle)\{1\} \stackrel{\partial}{\longrightarrow} \mathcal{H}(\asymp)\{2+3 \omega\}[1+\omega] \stackrel{i n_{*}}{\longrightarrow} \mathcal{H}(\underset{+}{\nwarrow}) \stackrel{p_{*}}{\longrightarrow} \mathcal{H}()()\{1\} \longrightarrow \cdots \\
& \cdots \longrightarrow \mathcal{H}(\rangle)\{-1\} \stackrel{i n_{*}}{\longrightarrow} \mathcal{H}\left(\mathbb{X}^{p_{*}} \stackrel{p_{*}}{\longrightarrow} \mathcal{H}(\asymp)\{1+3 \omega\}[\omega] \stackrel{\partial}{\longrightarrow} \mathcal{H}()()\{-1\} \longrightarrow \cdots\right.
\end{aligned}
$$

where $i n_{*}$ and $p_{*}$ are homogeneous and $\partial$ is the connecting differential and has bidegree $(1,0)$.

Remark. Long exact sequences (3.4) work equally well over any ring $R$ and for every version of the Khovanov homology, including the odd one (see [ORS]). This is both a blessing and a curse. On one hand, this means that all of the properties of the even Khovanov homology that are proved using these long exact sequences (and most of them are) hold automatically true for the odd Khovanov homology as well. On the other hand, this makes it very hard to find explanations to many differences between these homology theories.

\section{Applications of the Khovanov homology}

In this section we collect some of the more prominent applications of the Khovanov homology theories. This list is by no means complete and is chosen to provide the reader with a broader view on the type of problems that can be solved with a help of the Khovanov homology. We make a special effort to compare the performance of different versions of the homology, where applicable.

4.1. Rasmussen invariant and bounds on the slice genus. One of the most important applications of the Khovanov's construction so far was obtained by Jacob Rasmussen in 2004. In [Ra2] he used the structure of the Lee spectral sequence to define a new invariant of knots that gives a lower bound on the slice genus. More specifically, for a knot $L$, its Rasmussen invariant $s(L)$ is defined as the mean $q$-grading of the two copies of $\mathbb{Q}$ that remain in the homological grading 0 
of the limiting term of the Lee spectral sequence, see Theorem 3.2.B. Since the $q$-gradings of these $\mathbb{Q}$ 's are odd and are different by 2 , the Rasmussen invariant is an even integer.

4.1.A. Theorem (Rasmussen [Ra2]). Let $L$ be a knot and $s(L)$ its Rasmussen invariant. Then

- $|s(L)| \leq 2 g_{s}(L)$, where $g_{s}(L)$ is the slice genus of $L$, that is, the smallest possible genus of a smoothly embedded surface in the 4-ball $D^{4}$ that has $L \subset S^{3}=\partial D^{4}$ as its boundary;

- $s(L)=\sigma(L)$ for alternating $L$, where $\sigma(L)$ is the signature of $L$;

- $s(L)=2 g_{s}(L)=2 g(L)$ for a knot $L$ that possesses a planar diagram with positive crossings only, where $g(L)$ is the genus of $L$;

- if knots $L_{-}$and $L_{+}$have diagrams that are different at a single crossing in such a way that this crossing is negative in $L_{-}$and positive in $L_{+}$, then $s\left(L_{-}\right) \leq s\left(L_{+}\right) \leq s\left(L_{-}\right)+2$.

4.1.B. Corollary. $s\left(T_{p, q}\right)=(p-1)(q-1)$ for $p, q>0$, where $T_{p, q}$ is the $(p, q)$ torus knot. This implies the Milnor Conjecture, first proved by Kronheimer and Mrowka in 1993 using the gauge theory [KM1]. This conjecture states that the slice genus (and, hence, the genus) of $T_{p, q}$ equals $\frac{1}{2}((p-1)(q-1))$. The upper bound on the slice genus is straightforward, so the lower bound provided by the Rasmussen invariant is sharp.

Remark. Although the Rasmussen invariant was originally defined for knots only, its definition was later extended to the case of links by Anna Beliakova and Stephan Wehrli $[\mathrm{BW}]$.

The Rasmussen invariant can be used to search for knots that are topologically locally-flatly slice but are not smoothly slice (see [Sh3]). A knot is slice if its slice genus is 0 . Theorem 4.1.A implies that knots with non-trivial Rasmussen invariant are not smoothly slice. On the other hand, it was proved by Freedman $[\mathrm{F}]$ that knots with Alexander polynomial 1 are topologically locally-flatly slice. There are 82 knots with up to 16 crossings that possess these two properties [Sh3]. Each such knot gives rise to a family of exotic $\mathbb{R}^{4}$ [GS, Exercise 9.4.23]. It is worth noticing that most of these 82 examples were not previously known.

The Rasmussen invariant was also used [P,Sh3] to deduce the combinatorial proof of the Slice-Bennequin Inequality. This inequality states that

$$
g_{s}(\widehat{\beta}) \leq \frac{1}{2}(w(\beta)-k+1),
$$

where $\beta$ is a braid on $k$ strands with the closure $\widehat{\beta}$ and $w(\beta)$ is its writhe number. The Slice-Bennequin Inequality provides one of the upper bounds for the ThurstonBennequin number of Legendrian links (see below). It was originally proved by Lee Rudolph $[\mathrm{Ru}]$ using the gauge theory. The approach via the Rasmussen invariant and Khovanov homology avoids gauge theory and symplectic Floer theory and results in a purely combinatorial proof.

Remark. Since the Rasmussen's construction relies on the existence and convergence of the Lee spectral sequence, the Rasmussen invariant can only be defined for the even non-reduced Khovanov homology. In fact, a knot might not have any rational homology in the homological grading 0 of the odd Khovanov homology at all, see Figure 12. 
4.2. Bounds on the Thurston-Bennequin number. Another useful applications of the Khovanov homology is in finding upper bounds on the ThurstonBennequin number of Legendrian links. Consider $\mathbb{R}^{3}$ equipped with the standard contact structure $d z-y d x$. A link $K \subset \mathbb{R}^{3}$ is said to be Legendrian if it is everywhere tangent to the 2-dimensional plane distribution defined as the kernel of this 1-form. Given a Legendrian link $K$, one defines its Thurston-Bennequin number, $t b(K)$, as the linking number of $K$ with its push-off $K^{\prime}$ obtained using a vector field that is tangent to the contact planes but orthogonal to the tangent vector field of $K$. Roughly speaking, $t b(K)$ measures the framing of the contact plane field around $K$. It is well-known that the TB-number can be made arbitrarily small within the same class of topological links via stabilization, but is bounded from above.

4.2.A. Definition. For a given topological link $L$, let $\overline{t b}(L)$, the TB-bound of $L$, be the maximal possible TB-number among all the Legendrian representatives of $L$. In other words, $\overline{t b}(L)=\max _{K}\{t b(K)\}$, where $K$ runs over all the Legendrian links in $\mathbb{R}^{3}$ that are topologically isotopic to $L$.

Finding TB-bounds for links attracts considerable interest lately, since they can be used to demonstrate that certain contact structures on $\mathbb{R}^{3}$ are not isomorphic to the standard one. Such bounds can be obtained from the Bennequin and SliceBennequin inequalities, degrees of HOMFLY-PT and Kauffman polynomials, Knot Floer homology, and so on (see $[\mathrm{Ng}]$ for more details). The TB-bound coming from the Kauffman polynomial is usually one of the strongest, since most of the others incorporate another invariant of Legendrian links, the rotation number, into the inequality. In $[\mathrm{Ng}]$, Lenhard $\mathrm{Ng}$ used Khovanov homology to define a new bound on the TB-number.

4.2.B. Theorem $(\mathrm{Ng}[\mathrm{Ng}])$. Let $L$ be an oriented link. Then

$$
\overline{t b}(L) \leq \min \left\{k \mid \bigoplus_{j-i=k} \mathcal{H}^{i, j}(L ; R) \neq 0\right\} .
$$

Moreover, this bound is sharp for alternating links.

This Khovanov bound on the TB-number is often better than those that were known before. There are only two prime knots with up to 13 crossings for which the Khovanov bound is worse than the one coming from the Kauffman polynomial $[\mathrm{Ng}]$. There are 45 such knots with at most 15 crossings.

4.2.C. Example. Figure 11 shows computations of the Khovanov TB-bound for the $(4,-5)$-torus knot. The Khovanov homology groups in (4.2) can be used over any ring $R$, and this example shows that the bound coming from the integral homology is sometimes better than the one from the rational one, due to a strategically placed torsion. It is interesting to note that the integral Khovanov bound of -20 is computed incorrectly in $[\mathrm{Ng}]$. In particular, this was one of the cases where $\mathrm{Ng}$ thought that the Kauffman polynomial provides a better one. In fact, the TB-bound of -20 is sharp for this knot.

The proof of Theorem 4.2. B is based on the long exact sequences (3.4) and, hence, can be applied verbatim to the reduced as well as odd Khovanov homology. 


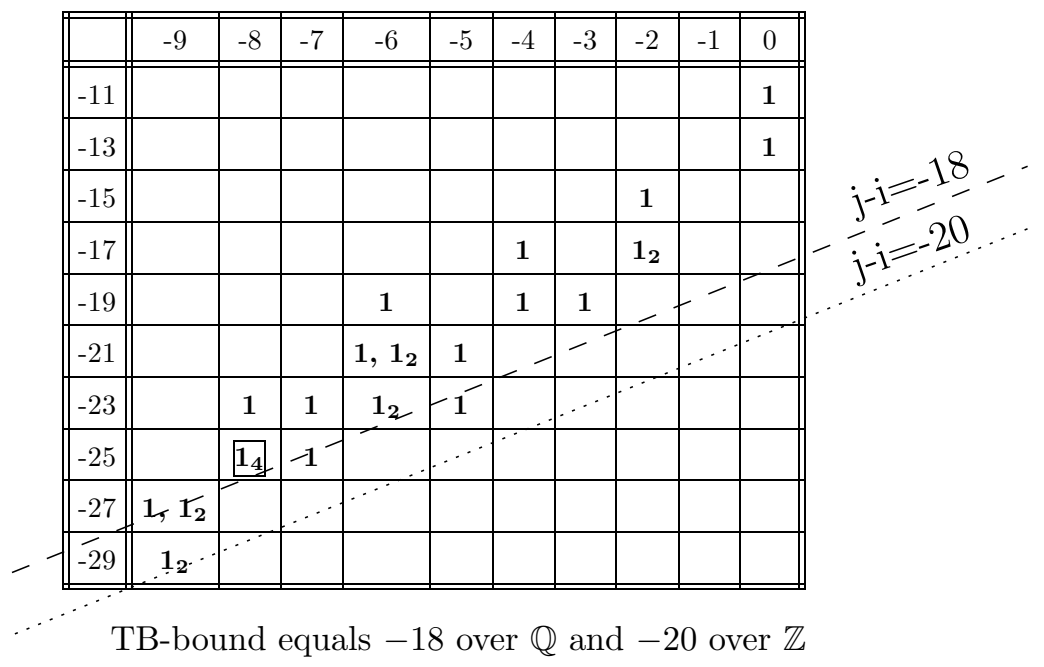

FiguRE 11. Khovanov TB-bound for the $(4,-5)$-torus knot

By making appropriate adjustments to the grading, we immediately get [Sh4] that

$$
\begin{aligned}
& \overline{t b}(L) \leq-1+\min \left\{k \mid \bigoplus_{j-i=k} \widetilde{\mathcal{H}}^{i, j}(L ; R) \neq 0\right\} \\
& \overline{t b}(L) \leq-1+\min \left\{k \mid \bigoplus_{j-i=k} \widetilde{\mathcal{H}}_{\text {odd }}^{i, j}(L ; R) \neq 0\right\} .
\end{aligned}
$$

As it turns out, the odd Khovanov TB-bound is often better than the even one. In fact, computations performed in [Sh4] show that the odd Khovanov homology provide the best upper bound on the TB-number among all currently known ones for all prime knots with at most 15 crossings. In particular, the odd Khovanov TB-bound equals the Kauffman one on all the 45 knots with at most 15 crossings where the latter is better than the even Khovanov TB-bound.

4.2.D. Example. Odd Khovanov TB-bound is better than the even one and equals to the Kauffman one for the knot $12_{475}^{n}$, as shown in Figure 12.

4.3. Finding quasi-alternating knots. Quasi-alternating links were introduced by Ozsváth and Szabó in [OS3] as a way to generalize the class of alternating links.

4.3.A. Definition. The class $\mathcal{Q}$ of quasi-alternating links is the smallest set of links such that

- the unknot belongs to $\mathcal{Q}$;

- if a link $L$ has a planar diagram $D$ such that the two resolutions of this diagram at one crossing represent two links, $L_{0}$ and $L_{1}$, with the properties that $L_{0}, L_{1} \in \mathcal{Q}$ and $\operatorname{det}(L)=\operatorname{det}\left(L_{0}\right)+\operatorname{det}\left(L_{1}\right)$, then $L \in \mathcal{Q}$ as well.

Remark. It is well-known that all non-split alternating links are quasi-alternating.

The main motivation for studying quasi-alternating links is the fact that the double branched covers of $S^{3}$ along such links are so-called $L$-spaces. A 3-manifold $M$ is called an $L$-space if the order of its first homology group $H_{1}$ is finite and 


\begin{tabular}{|r||l|l|l|l|l|l|l|l||}
\hline \hline & 0 & 1 & 2 & 3 & 4 & 5 & 6 & 7 \\
\hline \hline 13 & & & & & & & & $\mathbf{1}$ \\
\hline 11 & & & & & & & & $\mathbf{1}_{\mathbf{2}}$ \\
\hline 9 & & & & & & $\mathbf{1}$ & $\mathbf{1}$ & \\
\hline 7 & & & & & $\mathbf{1}$ & $\mathbf{1}_{\mathbf{2}}$ & & \\
\hline 5 & & & & & $\mathbf{1}, \mathbf{1}_{\mathbf{2}}$ & & & \\
\hline 3 & & & $\mathbf{1}$ & $\mathbf{1}$ & & & & \\
\hline 1 & $\mathbf{1}$ & & $\mathbf{1}_{\mathbf{2}}$ & & & & & \\
\hline-1 & $\mathbf{1}$ & $\mathbf{1}$ & & & & & & \\
\hline \hline
\end{tabular}

(even) Khovanov homology

TB-bound equals -2

\begin{tabular}{||r||r|r|r|r|r|r|r|r||}
\hline \hline & 0 & 1 & 2 & 3 & 4 & 5 & 6 & 7 \\
\hline \hline 12 & & & & & & & & $\mathbf{1}$ \\
\hline 10 & & & & & & & $\mathbf{1}$ & \\
\hline 8 & & & & & & $\mathbf{1}$ & & \\
\hline 6 & & & & & $\mathbf{2}$ & & & \\
\hline 4 & & & & $\mathbf{1}$ & & & & \\
\hline 2 & & & $\mathbf{1}, \underline{\mathbf{1}_{3}}$ & & & & & \\
\hline 0 & & $\mathbf{1}_{\mathbf{8}}$ & & & & & & \\
\hline-2 & $\mathbf{1}_{\mathbf{3}}$ & & & & & & & \\
\hline \hline
\end{tabular}

odd reduced Khovanov homology

TB-bound equals -3

Figure 12. Khovanov TB-bounds for the knot $12_{475}^{n}$

equals the rank of the Heegaard-Floer homology of $M$ (see [OS3]). Unfortunately, due to the recursive style of Definition 4.3.A, it is often highly non-trivial to prove that a given link is quasi-alternating. It is equally challenging to show that it is not.

To determine that a link is not quasi-alternating, one usually employs the fact that such links have homologically thin Khovanov homology over $\mathbb{Z}$ and Knot Floer homology over $\mathbb{Z}_{2}$ (see $[\mathrm{MO}]$ ). Thus, $\mathbb{Z} \mathrm{H}$-thick knots are not quasi-alternating. There are 12 such knots with up to 10 crossings. Most of the others can be shown to be quasi-alternating by various constructions. After the work of Champanerkar and Kofman $[\mathrm{ChK}]$, there were only two knots left, $9_{46}$ and $10_{140}$, for which it was not known whether they are quasi-alternating or not. Both of them have homologically thin Khovanov and Knot Floer homology.

As it turns out, odd Khovanov homology is much better at detecting quasialternating knots. The proof of the fact that such knots are $\mathbb{Z H}$-thin is based on the long exact sequences (3.4) and, therefore, can be applied verbatim to the odd homology as well [ORS]. Computations show [Sh4] that the knots $9_{46}$ and $10_{140}$ have homologically thick odd Khovanov homology and, hence, are not quasialternating, see Figures 13 and 14.

Remark. It is worth mentioning that the knots $9_{46}$ and $10_{140}$ are $(3,3,-3)$ - and $(3,4,-3)$-pretzel knots, respectively (see Figure 15 for the definition). Computations show that $(n, n,-n)$ - and $(n, n+1,-n)$-pretzel links for $n \leq 6$ all have torsion of order $n$ outside of the main diagonal that supports the free part of the homology. This suggest a certain $n$-fold symmetry on the odd Khovanov chain complexes for these pretzel links that cannot be explained by the construction.

Remark. Joshua Greene has recently determined [Gr] all quasi-alternating pretzel links by considering 4-manifolds that are bounded by the branched double covers of the links. In particular, he found several knots that are not quasi-alternating, yet both H-thin and OH-thin. The smallest such knot is $11_{50}^{n}$.

4.4. Detection of the unknot. It was recently showed by Kronheimer and Mrowka [KM2] that Khovanov homology detects the unknot. More specifically, 


\begin{tabular}{|r||l|l|l|l|l|l|l||}
\hline \hline & -6 & -5 & -4 & -3 & -2 & -1 & 0 \\
\hline \hline 0 & & & & & & & $\mathbf{2}$ \\
\hline-2 & & & & & & $\mathbf{1}$ & \\
\hline-4 & & & & & $\mathbf{1}$ & & \\
\hline-6 & & & & $\mathbf{2}$ & & & \\
\hline-8 & & & $\mathbf{1}$ & & & & \\
\hline-10 & & $\mathbf{1}$ & & & & & \\
\hline-12 & $\mathbf{1}$ & & & & & & \\
\hline \hline
\end{tabular}

(even) reduced Khovanov homology

\begin{tabular}{||r||c|c|c|c|c|c|c||}
\hline \hline & -6 & -5 & -4 & -3 & -2 & -1 & 0 \\
\hline \hline 0 & & & & & & & $\mathbf{2}$ \\
\hline-2 & & & & & & $\mathbf{1}$ & $\mathbf{\mathbf { 1 } _ { 3 }}$ \\
\hline-4 & & & & & $\mathbf{1}$ & & \\
\hline-6 & & & & $\mathbf{2}$ & & & \\
\hline-8 & & & $\mathbf{1}$ & & & & \\
\hline-10 & & $\mathbf{1}$ & & & & & \\
\hline-12 & $\mathbf{1}$ & & & & & & \\
\hline \hline
\end{tabular}

odd reduced Khovanov homology

Figure 13. Khovanov homology of $9_{46}$, the $(3,3,-3)$-pretzel knot

\begin{tabular}{||r||r|r|r|r|r|r|r|r||}
\hline \hline & -7 & -6 & -5 & -4 & -3 & -2 & -1 & 0 \\
\hline \hline 0 & & & & & & & & $\mathbf{1}$ \\
\hline-2 & & & & & & & $\mathbf{1}$ & \\
\hline-4 & & & & & & $\mathbf{1}$ & & \\
\hline-6 & & & & & $\mathbf{1}$ & & & \\
\hline-8 & & & & $\mathbf{2}$ & & & & \\
\hline-10 & & & $\mathbf{1}$ & & & & & \\
\hline-12 & & $\mathbf{1}$ & & & & & & \\
\hline-14 & $\mathbf{1}$ & & & & & & & \\
\hline \hline
\end{tabular}

(even) reduced Khovanov homology

\begin{tabular}{||r||r|r|r|r|r|r|r|r||}
\hline \hline & -7 & -6 & -5 & -4 & -3 & -2 & -1 & 0 \\
\hline \hline 0 & & & & & & & & $\mathbf{1}$ \\
\hline-2 & & & & & & & $\mathbf{1}$ & \\
\hline-4 & & & & & & $\mathbf{1}$ & $\mathbf{1}_{\mathbf{3}}$ & \\
\hline-6 & & & & & $\mathbf{1}$ & & & \\
\hline-8 & & & & $\mathbf{2}$ & & & & \\
\hline-10 & & & $\mathbf{1}$ & & & & & \\
\hline-12 & & $\mathbf{1}$ & & & & & & \\
\hline-14 & $\mathbf{1}$ & & & & & & & \\
\hline \hline
\end{tabular}

odd reduced Khovanov homology

Figure 14. Khovanov homology of $10_{140}$, the $(3,4,-3)$-pretzel knot
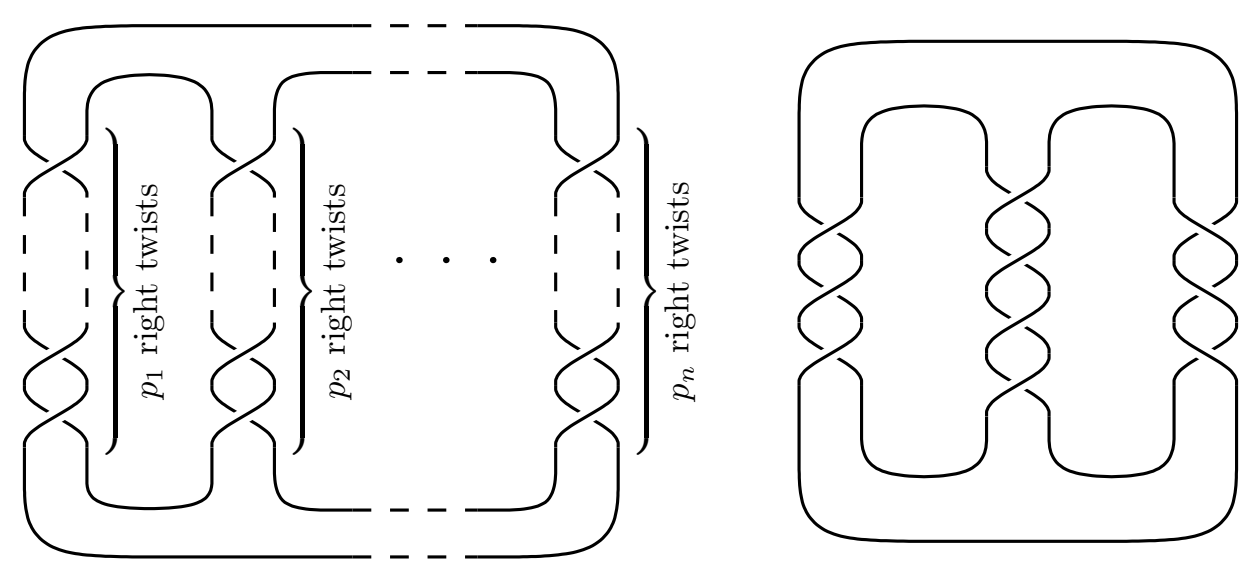

Figure 15. $\left(p_{1}, p_{2}, \ldots, p_{n}\right)$-pretzel link and $(3,4,-3)$-pretzel knot $10_{140}$ 
they proved that a knot is the unknot if and only if its reduced Khovanov homology has rank 1. This development is a major step towards proving a long-standing conjecture that the Jones polynomial itself detects the unknot.

\section{REFERENCES}

[AKh] M. Asaeda and M. Khovanov, Notes on link homology, in Low dimensional topology, 139-195, IAS/Park City Math. Ser., 15, Amer. Math. Soc., Providence, RI, 2009; arXiv:0804.1279.

[BN1] D. Bar-Natan, On Khovanov's categorification of the Jones polynomial, Alg. Geom. Top., 2 (2002) 337-370; arXiv:math.QA/0201043.

[BN2] D. Bar-Natan, Fast Khovanov Homology Computations, J. Knot Th. and Ramif. 16 (2007), no. 3, 243-255; arXiv:math.GT/0606318.

[BNG] D. Bar-Natan and J. Green, JavaKh - a fast program for computing Khovanov homology, part of the KnotTheory' Mathematica Package, http://katlas.math.utoronto.ca/wiki/Khovanov_Homology

[BW] A. Beliakova and S. Wehrli, Categorification of the colored Jones polynomial and Rasmussen invariant of links, Canad. J. Math. 60 (2008), no. 6, 1240-1266; arXiv:math.GT/0510382.

[B] J. Bloom, Odd Khovanov homology is mutation invariant, Math. Res. Lett. 17 (2010), no. 1, 1-10; arXiv:0903.3746.

[ChK] A. Champanerkar and I. Kofman, Twisting quasi-alternating links, Proc. Amer. Math. Soc. 137 (2009), 2451-2458; arXiv:0712.2590.

[DGShT] N. Dunfield, S. Garoufalidis, A. Shumakovitch, and M. Thistlethwaite, Behavior of knot invariants under genus 2 mutation, New York J. Math. 16 (2010), 99-123; arXiv:math.GT/0607258.

[F] M. Freedman, A surgery sequence in dimension four; the relations with knot concordance, Invent. Math. 68 (1982), no. 2, 195-226.

[GS] R. Gompf and A. Stipsicz, 4-manifolds and Kirby calculus, Graduate Studies in Mathematics 20, American Mathematical Society, Providence, RI, 1999.

[Gr] J. Greene, Homologically thin, non-quasi-alternating links, Math. Res. Lett. 17 (2010), no. 1, 39-49; arXiv:0906.2222.

[HTh] J. Hoste and M. Thistlethwaite, Knotscape - a program for studying knot theory and providing convenient access to tables of knots, http://www. math.utk.edu/ morwen/knotscape.html

[J] V. Jones, A polynomial invariant for knots via von Neumann algebras, Bull. Amer. Math. Soc. 12 (1985), 103-111.

[K] L. Kauffman, State models and the Jones polynomial, Topology 26 (1987), no. 3, 395-407.

[Kh1] M. Khovanov, A categorification of the Jones polynomial, Duke Math. J. 101 (2000), no. 3, 359-426; arXiv:math.QA/9908171.

[Kh2] M. Khovanov, Patterns in knot cohomology I, Experiment. Math. 12 (2003), no. 3, 365-374; arXiv:math.QA/0201306.

[Kh3] M. Khovanov, Categorifications of the colored Jones polynomial, J. Knot Th. Ramif. 14 (2005), no. 1, 111-130; arXiv:math.QA/0302060.

[Kh4] M. Khovanov, sl(3) link homology I, Algebr. Geom. Topol. 4 (2004), 1045-1081; arXiv:math.QA/0304375.

[Kh5] M. Khovanov, Link homology and Frobenius extensions, Fundamenta Mathematicae, 190 (2006), 179-190; arXiv:math.QA/0411447.

[Kh6] M. Khovanov, Link homology and categorification, ICM-2006, Madrid, Vol. II, 989-999, Eur. Math. Soc., Zürich, 2006; arXiv:math/0605339.

[KhR1] M. Khovanov and L. Rozansky, Matrix factorizations and link homology, Fund. Math. 199 (2008), no. 1, 1-91; arXiv:math.QA/0401268.

[KhR2] M. Khovanov and L. Rozansky, Matrix factorizations and link homology II, Geom. Topol. 12 (2008), no. 3, 1387-1425; arXiv:math.QA/0505056.

[KhR3] M. Khovanov and L. Rozansky, Virtual crossings, convolutions and a categorification of the $S O(2 N)$ Kauffman polynomial, J. Gökova Geom. Topol. GGT 1 (2007), 116-214; arXiv:math/0701333. 
[KM1] P. Kronheimer and T. Mrowka, Gauge Theory for Embedded Surfaces I, Topology 32 (1993), 773-826.

[KM2] P. Kronheimer and T. Mrowka, Khovanov homology is an unknot-detector, arXiv:1005.4346.

[L] E. S. Lee, An endomorphism of the Khovanov invariant, Adv. Math. 197 (2005), no. 2, 554-586; arXiv:math.GT/0210213.

[MO] C. Manolescu, P. Ozsváth, On the Khovanov and knot Floer homologies of quasi-alternating links in Proc. of Gökova Geometry-Topology Conference 2007, 60-81, Gökova Geometry/Topology Conference (GGT), Gökova, 2008; arXiv:0708.3249.

[Ng] L. Ng, A Legendrian Thurston-Bennequin bound from Khovanov homology, Algebr. Geom. Topol. 5 (2005) 1637-1653; arXiv:math.GT/0508649.

[ORS] P. Ozsváth, J. Rasmussen, and Z. Szabó, Odd Khovanov homology, arXiv:0710.4300.

[OS1] P. Ozsváth and Z. Szabó, Holomorphic disks and topological invariants for closed three-manifolds, Ann. of Math. 159 (2004) 1027-1158; arXiv:math.SG/0101206.

[OS2] P. Ozsváth and Z. Szabó, Holomorphic disks and knot invariants, Adv. Math. 186 (2004), no. 1, 58-116; arXiv:math.GT/0209056.

[OS3] P. Ozsváth and Z. Szabó, On the Heegaard Floer homology of branched double-covers, Adv. Math. 194 (2005), no. 1, 1-33; arXiv:math.GT/0309170.

[P] O. Plamenevskaya, Transverse knots and Khovanov homology, Math. Res. Lett. 13 (2006), no. 4, 571-586; arXiv:math.GT/0412184.

[Ra1] J. Rasmussen, Floer homology and knot complements, Ph.D. Thesis, Harvard U.; arXiv:math.GT/0306378.

[Ra2] J. Rasmussen, Khovanov homology and the slice genus, to appear in Invent. Math.; arXiv:math.GT/0402131.

[Ra3] J. Rasmussen, Knot polynomials and knot homologies, Geometry and Topology of Manifolds (Boden et al eds.), Fields Institute Communications 47 (2005) 261-280, AMS; arXiv:math.GT/0504045.

[Ro] D. Rolfsen, Knots and Links, Publish or Perish, Mathematics Lecture Series 7, Wilmington 1976.

[Ru] L. Rudolph, Quasipositivity as an obstruction to sliceness, Bull Amer. Math. Soc. (N.S.) 29 (1993), no. 1, 51-59; arXiv:math.GT/9307233.

[Sh1] A. Shumakovitch, KhoHo - a program for computing and studying Khovanov homology, http://www.geometrie.ch/KhoHo

[Sh2] A. Shumakovitch, Torsion of the Khovanov homology, arXiv:math.GT/0405474; to appear in Fund. Math.

[Sh3] A. Shumakovitch, Rasmussen invariant, Slice-Bennequin inequality, and sliceness of knots, J. Knot Th. and Ramif., 16 (2007), no. 10, 1403-1412; arXiv:math.GT/0411643.

[Sh4] A. Shumakovitch, Patterns in odd Khovanov homology, to appear in J. Knot Th. and Ramif.

[T] P. Turner, Calculating Bar-Natan's characteristic two Khovanov homology, J. Knot Th. Ramif. 15 (2006), no. 10, 1335-1356; arXiv:math.GT/0411225.

[V] O. Viro, Khovanov homology, its definitions and ramifications, Fund. Math. 184 (2004), 317-342; arXiv:math.GT/0202199.

[W1] S. Wehrli, Khovanov Homology and Conway Mutation, arXiv:math/0301312.

[W2] S. Wehrli, Mutation invariance of Khovanov homology over $\mathbb{F}_{2}$, Quantum Topol. 1 (2010), no. 2, 111-128; arXiv:0904.3401.

[Wu] H. Wu, A colored $\mathfrak{s l}(N)$-homology for links in $S^{3}$, arXiv:0907.0695.

$[\mathrm{Y}] \quad \mathrm{Y}$. Yonezawa, Quantum $\left(\mathfrak{s l}_{n}, \wedge V_{n}\right)$ link invariant and matrix factorizations, arXiv:0906.0220.

Department of Mathematics, The George Washington University, Monroe Hall 2115 G St. NW, Washington, DC 20052, U.S.A.

E-mail address: Shurik@gwu.edu 